Jaime Andrés Vargas Vives* Juan Sergio Cruz Merchán**

Pontificia Universidad Javeriana, Bogotá, Colombia.

Recibido: 30 de marzo de 2014

Concepto de evaluación: 9 de junio de 2014

Aprobado: 27 de noviembre de 2014

Artículo de investigación (C) 2015 Universidad Católica de Colombia. Facultad de Ciencias Económicas y Administrativas. Todos los derechos reservados
* Magíster en Administración. Especialista en Finanzas Corporativas. Ingeniero industrial. Profesor e investigador de

la Pontificia Universidad Javeriana, Bogotá, Colombia. Dirección de correspondencia: calle $64 \mathrm{~N} .^{\circ}$

2-55, torre 1, apto 202, Bogotá, Colombia. Correo electrónico: jvargasv@javeriana.edu.co

** Magíster en Política Internacional en Comunidades Europeas. Especialista en Finanzas. Profesor de la Pontificia Universidad Javeriana, Bogotá, Colombia. Correo electrónico: jcruz2009@hotmail.com
Finanz. polit. econ., ISSN: 2248-6046, Vol. 7, No. 1, enero-junio, 2015, pp. 55-82 http://dx.doi.org/10.14718/revfinanzpolitecon.2015.7.1.3

\section{Generación del valor a partir de la gerencia del riesgo sistemático}

\section{RESUMEN}

Este artículo tiene como propósito profundizar en cómo se conceptualiza la teoría, la aplicación del riesgo, su gestión y valor. Se demostrará cómo los modelos de gerencia del riesgo pueden crear valor a través de la reducción de la tasa de descuento de los flujos de valoración del activo subyacente. La investigación planteará tres modelos de derivados reales: derivados de ventas, derivados de costos y derivados de EBIT. El propósito de estos derivados reales se centrará en la maximización del valor del activo a través de estrategias de reducción del riesgo sistemático (beta). En síntesis, los resultados de esta investigación contradicen uno de los postulados centrales de la gerencia moderna, liderado por C. W. Smith en su bien conocido artículo "Corporative risk management".

Palabras clave: beta, riesgo sistemático, activo subyacente, valor, swap, resultado de explotación (EBIT), utilidad operacional.

JEL: D81, G12, G32, L97

\section{Creating Value through Systematic Risk Management}

\section{ABSTRACT}

This article explores the concept of risk in terms of its theory, application, management and value. The study shows how risk management models can create value by reducing the discount rate of valuation flows of the underlying asset Three models of real derivatives are put forward: sales derivatives, cost derivatives, and EBIT derivatives. These real derivatives focus on maximizing asset value through systematic risk (beta) reduction strategies. In summary, the results of this study contradict one of the central principles of modern management, put forward by C. W. Smith in his well-known article, "Corporate risk management".

Keywords: beta, systematic risk, underlying asset, value, swap, Earnings Before Interest and Taxes (EBIT), operating utility. 


\section{Geração do valor a partir da gerência do risco sistemático}

\section{RESUMO}

Este artigo tem como objetivo aprofundar em como se conceituam a teoria, a aplicação do risco, sua gestão e valor. Demonstra-se como os modelos de gerência do risco podem criar valor por meio da redução da taxa de desconto dos fluxos de avaliação do ativo subjacente. Esta pesquisa propõe três modelos de derivados reais: derivados de vendas, derivados de custos e derivados de EBIT. O propósito desses derivados reais se centralizará na maximização do valor do ativo por meio de estratégias de redução do risco sistemático (beta). Em síntese, os resultados desta pesquisa contradizem uma das premissas centrais da gerência moderna, liderada por C. W. Smith em seu conhecido artigo "Corporative risk management".

Palavras-chave: beta, risco sistemático, ativo subjacente, valor, swap, resultado de exploração (EBIT), utilidade operacional. 


\section{INTRODUCCIÓN}

Las teorías en los ámbitos económicos y financieros tienen que evolucionar, y al igual que otras teorías en distintos campos académicos, siempre están sujetas a crítica, a cambios en el tiempo y controversia. Los avances en el último siglo han sido significativos: desde la teoría de Keynes (1936) de demanda agregada y su relación con el nivel de empleo e ingresos, Samuelson (1947) y su desarrollo de las teorías económicas del bienestar y sus aportes a la economía keynesiana, hasta las teorías financieras más recientes como las de Modigliani y Miller (1958) de costo de capital, Markowitz (1952) y Sharpe (1964) con las teorías de portafolio y el modelo CAPM (valoración del precio de los activos financieros), y finalmente por mencionar están los modelos de Black y Scholes (1972) y Merton (1973) para la valoración de contratos derivados.

Adicionales a los grandes avances en el campo económico y financiero ya mencionados, se encuentran aquellos del matemático y economista Forbes Nash (1950), cuyos desarrollos en el área de teoría de juegos han tenido grandes aplicaciones en dichos campos y, en efecto, servirán como soporte para el presente trabajo. Sin embargo, genera gran preocupación para el ámbito actual el hecho de que a partir de 1973 no se hayan producido grandes aportes en estos campos académicos.

Los teóricos se han dedicado a contradecirse o a tratar de perfeccionar modelos que ya existen, sin poder llegar a un consenso general. Recientes desarrollos como la teoría de agencia, las teorías de información asimétrica, los modelos para volatilidad no constante (ARCH), las teorías de cointegración, la teoría de opciones reales y la teoría del diseño de mecanismos han sobresalido como los aportes más significativos durante los últimos treinta años. Muchas de estas contribuciones han sido relevantes, aunque siguen siendo marginales en importancia al medirlas con las teorías que se construyeron entre 1936 y 1973. La teoría tradicional de la gerencia del riesgo en realidad solo se ha dedicado a la reducción del riesgo como tal, para poder minimizar las pérdidas que este pueda generar. Este aspecto es tan solo una cara de la moneda y se centra en el lado izquierdo de la curva del riesgo: el downside risk (riesgo a la baja).

Así, el miedo por la probabilidad de pérdida ha llevado a que los inversionistas solo se dediquen a gerenciar el downside risk. Sin embargo, existe otro lado del riesgo que puede ser igual o aun más importante en el desarrollo de una gerencia del riesgo: el upside risk (riesgo al alza). Según Damodaran (2008), este último referencia la gerencia estratégica del riesgo con el objetivo de la creación del valor. Por ello, este trabajo lidiará con el riesgo como problema fundamental y se centrará en un adecuado manejo de este que cubra tanto el downside como el upside del riesgo, orientado al objetivo primordial de la gerencia: la creación de valor para los accionistas.

En la teoría económica financiera, el objetivo final es la maximización del bienestar. Este, que alguna vez se medía a través del concepto de la utilidad, hoy en día se define a través del concepto económico del valor. La maximización de valor siempre ha sido una utopía que ha intrigado al humano, sea este el valor de un solo agente o el valor de una sociedad, aun cuando, en términos de la teoría de juegos, la decisión de maximizar al valor de una sociedad maximiza los valores de los agentes, a través de las decisiones conjuntas orientadas hacia el beneficio colectivo.

Las teorías financieras han ayudado a determinar funciones desde las cuales puede modelarse el valor de un activo; sin embargo, y al igual que muchas otras teorías de este tipo, aquellas tienen deficiencias en sus aplicaciones o en los supuestos que exigen cumplir para su práctica. Más allá de la falta de un consenso general sobre lo adecuados que son estos modelos de valoración, se encuentra la subjetividad de aquel que los utiliza. No obstante, en sus versiones más simples, los modelos tienen una conceptualización teórica adecuada que funciona, y los problemas subyacen en las aplicaciones de dichos modelos cuando se quiebran los supuestos. Para ello, es necesario hallar una forma de relajar los supuestos, para tener en cuenta su aplicabilidad. A partir de lo anterior, 
se planteará la siguiente función de valor en su forma más simple:

Valor Activo $_{i}=f\left(\right.$ flujos $\left._{i}, T D_{i}\right)=\sum_{i=1}^{n} \frac{F C_{i}}{\left(1+T D_{i}\right)^{i}}$

En la versión más simple, el valor de un activo puede expresarse en función de dos variables: los flujos de caja proyectados de ese activo y las tasas de descuento correspondiente a la incertidumbre o el riesgo implícito en los flujos de caja proyectados.

Según lo anterior, el objetivo de este trabajo se basa en el desarrollo de la maximización del valor de un activo, a través de estrategias implementadas para gerenciar uno de los dos factores que afectan el valor de un activo: el riesgo. Esto se logrará a través de la creación de cinco modelos de gerencia de cobertura de riesgo que de ahora en adelante llamaremos modelos de derivados reales, que buscarán el máximo del valor, mediante la maximización de los flujos de caja esperados o la minimización de la tasa de descuento.

\section{MODELOS DE DERIVADOS REALES}

Una de las teorías más significativas en la economía financiera fue la propuesta por William F. Sharpe en 1964: el Capital Asset Pricing Model (CAPM), o modelo de valoración del precio de los activos financieros. A través de su planteamiento teórico, el autor concluyó que la noción de "riesgo", calculada a través de la varianza o la medida de dispersión de los posibles valores que podría tomar ese activo, podía diversificarse. Esto podía lograrse mediante inversiones en otros activos cuya correlación fuera menor a 0. Finalmente, y con el objetivo de minimizar este "riesgo", Sharpe (1964) concluyó que un inversionista racional terminaría invirtiendo en el máximo número de activos posibles y que este riesgo individual dejaría de ser importante, ya que podría diversificarse. A este riesgo fue al que él luego se refirió como riesgo diversificable. Así, a partir de la teoría de diversificación del portafolio, el riesgo diversificable deja de ser relevante; según Sharpe, el único componente que representa importancia es el riesgo no diversificable. De acuerdo con lo anterior, una de las implicaciones del modelo CAPM es que a los accionistas que se encuentren diversificados solamente debe importarles el componente de riesgo que no puede diversificarse, también conocido como riesgo sistemático, riesgo de mercado o riesgo beta.

Las teorías de costo de capital de Modigliani y Miller (1958) también soportan las proposiciones de Sharpe (1964). Una extensión de las anteriores afirmaciones es que al no ser relevantes los riesgos diversificables, las estrategias de cobertura que se implementen sobre estos no tendrán ningún efecto respecto al valor del activo subyacente. Por ello, lo único que sí sería relevante en la estructura de valor de un activo serían aquellas estrategias de cobertura del riesgo sistemático, no diversificable o de mercado. Sin embargo, nacen tres disyuntivas en este sentido: ¿por qué los instrumentos de cobertura existentes solo apuntan a cubrir riesgos diversificables?, ¿será que las coberturas del riesgo diversificable pueden crear valor?, ¿cómo pueden desarrollarse instrumentos de coberturas del riesgo no diversificable?

Cuando Sharpe propuso su teoría de CAPM, concluyó que el único riesgo relevante sería el no diversificable, como ya se mencionó. Los instrumentos derivados más comúnmente utilizados son los contratos forwards (a corto plazo), los contratos de futuros, los contratos swaps (de permuta financiera) y los contratos de opciones que se realizan sobre acciones, tasas de intereses, tasas de cambio y precios de commodities (materias primas), entre otros. Para un accionista, todos estos riesgos pueden ser considerados diversificables en el mercado: el riesgo de una acción puede diversificarse al invertir en un portafolio de acciones diversificado; el riesgo tasa de cambio puede hacerlo al invertir en un portafolio de monedas diversificado, y lo mismo puede decirse de los otros. Pereciese que existiera una contradicción: si el riesgo diversificable no resulta relevante, ¿para qué realizar coberturas sobre este?

Según Smith (1995), si las decisiones financieras, incluyendo las coberturas de riesgo, afectan el valor de una empresa, entonces deben hacerlo 
a través de impuestos, costos de transacción o políticas de inversión; es decir, sería posible que una cobertura sobre un riesgo diversificable creara valor a través de algún impacto en las tres variables mencionadas. Sin embargo, si estas tres son las únicas formas en que una cobertura de riesgo afecta el valor de un activo, entonces, ¿cómo una cobertura de un riesgo sistemático podría incidir en el valor de un activo? Clifford argumenta: "For risk management to increase the value of a widely held firm, if it does not reduce the firm's required rate of return, then it must increase the firms expected net cash flow" (citado en Smith y Stulz, 1985). Esto puede resumirse en la siguiente ecuación:

Valor Activo $_{i}=\sum_{i=1}^{n} \frac{F C_{i}}{\left(1+T D_{i}\right)^{i}}\left\{\begin{array}{l}\text { Efecto en el valor vía: } \\ \text {-Impuestos } \\ \text { - Costos de transacción } \\ \text {-Política de inversión }\end{array}\right.$

Sin embargo, de acuerdo con las proposiciones de este trabajo, es posible hacer coberturas del riesgo sistemático que afecten la volatilidad de los flujos de caja proyectados de un activo y, por ende, reduzcan su tasa de descuento sin ocasionar una disminución en los flujos de caja. Estas coberturas, si bien pueden pactarse a través de instrumentos financieros, también pueden plantearse a través de determinadas estrategias. De igual forma, el valor presente neto (VPN) del efecto de los derivados reales es mayor que el costo de transacción de los instrumentos financieros. Esto nos llevará a concluir que el efecto de una cobertura real puede disminuir y, aún más importante, tener un efecto sobre el beta de un activo sin afectar sus flujos de caja $y$, por ende, aumentar su valor.

El concepto del riesgo sistemático nació con Sharpe (1964), y según Damodaran (2002), el beta de una firma es determinado por tres variables en la compañía: a) el tipo de negocio o negocios, b) el grado de apalancamiento operativo y c) el grado de apalancamiento financiero. El tipo de negocio mide la sensibilidad de la empresa según condiciones macroeconómicas o del mercado; el grado de apalancamiento operativo es una función de la estructura de costos de la empresa y normalmente es determinado por la relación entre costos fijos y variables; la tercera variable que determina el valor de un beta es el grado de apalancamiento financiero. Para el propósito de nuestros primeros tres modelos, supondremos una empresa sin apalancamiento financiero (all-equity firm). A continuación se plantearán tres modelos de derivados reales sobre la estructura operacional de pérdidas y ganancias de un activo, que es base fundamental en la derivación de sus flujos de caja. El propósito de estos derivados reales se centrará sobre la maximización del valor del activo a través de estrategias de reducción del riesgo sistemático (beta).

\section{DERIVADO REAL SOBRE VENTAS}

Las ventas o los ingresos operacionales pueden caracterizarse como la razón de ser de cualquier empresa, sea esta de carácter industrial, comercializadora o de servicios. La teoría económica dice que la cantidad de ingresos generados por la empresa depende tanto del precio como de la cantidad vendida. De esta forma, la función de ventas puede resumirse así:

$S_{t}=$ función de ventas $=f(P, Q)$

$S_{t}=\sum_{i=1}^{N} P_{i t} Q_{i t}$

Donde,

$S_{t}=$ ventas en el momento $t$

$P_{i t}=$ precio del producto $i$ en el tiempo $t$

$Q_{i t}=$ cantidad vendida del producto $i$ en el tiempo $t$

$N=$ número de productos

De manera simplificada, puede concluirse que las ventas en sí como función dependen tan solo de dos variables: el precio de los productos que se comercializan, que puede referirse como activo subyacente, y la demanda de ese activo. Según la teoría microeconómica, la cantidad demandada de un bien depende de cuatro variables: el precio de 
ese bien $\left(P_{A}\right)$, la renta $(Y)$, el precio de los demás bienes $\left(P_{B}\right)$ y el gusto de los consumidores $(G)$. Lo anterior puede modelarse así:

$Q_{A}=f\left\{P_{A}, Y, P_{B}, G\right\}$

Al tomar como supuesto que existe un equilibrio perfecto, pueden igualarse las curvas de demanda y oferta para hallar el precio de equilibrio. La función de oferta de un producto depende del precio de ese bien $\left(P_{A}\right)$, el precio de los demás bienes $\left(P_{B}\right)$, los precios de los factores productivos $(r)$, la tecnología $(z)$ y los gustos o las preferencias de los productores $(H)$. A partir de lo anterior, es posible hallar un precio equilibrio en el mercado (gráfica 1 ).

Gráfica 1

Equilibrio entre oferta y demanda

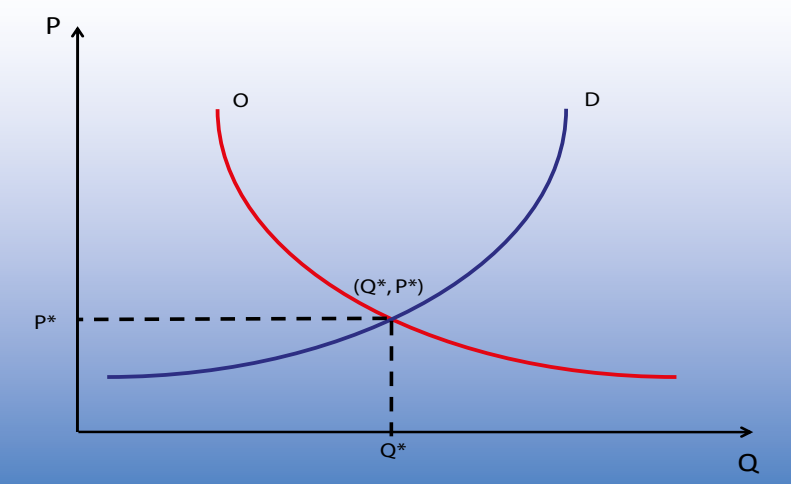

Fuente: elaboración de los autores.

Los anteriores dos factores: el precio del activo subyacente y la cantidad de ese producto demandado, reflejan el comportamiento de la primera variable a la cual se refiere Damodaran (2002) y que determina el beta de una firma: el tipo de negocio y la sensibilidad de la empresa según condiciones macroeconómicas. Estos pueden verse materializados en el comportamiento de su función de demanda y en los choques que tenga el precio ante factores del mercado. En su teoría de eficiencia de mercado (MEH), Fama (1970) argumentaba que, en sí misma, la competencia en el mercado para especular hacía que los precios incorporaran inmediatamente la información pública y disponible a la forma que más se asimilaba allí: la forma semifuerte. Esto llevaba a que fuera inútil cualquier mecanismo desarrollado con el propósito de predecir el precio futuro de un activo, ya que dicha información, siempre y cuando fuera pública, estaría contenida dentro del precio presente de dicho activo. De este modo, Fama (1970) concluyó que en un mercado eficiente, los precios de los activos se comportaban como una variable aleatoria, siguiendo una caminata también aleatoria:

$P_{t}=P_{t-1}+\varepsilon_{t} \rightarrow \varepsilon_{t} \sim N(0, \sigma), I I D$

Este modelo de caminata aleatoria se derivaba como una simplificación de un modelo de movimiento browniano, y el resultado de su proceso estocástico se conoce como proceso estocástico Wiener:

$\frac{\partial P}{P}=\mu \partial t+\sigma d z$

La teoría de Fama (1970) llevó a la conclusión de que el mejor estimativo del precio futuro de un activo es el precio de hoy, y que los choques que se produzcan en el activo no pueden predecirse, tienen como media 0 y se distribuyen normalmente. Más allá, podría determinarse que la función de demanda es el segundo gran factor que define qué tan sensible es un activo en el mercado y su relación con la oferta. De acuerdo con lo anterior, puede concluirse que si pudiese crearse un instrumento que llegase a realizar una cobertura sobre los factores que afectan la sensibilidad de un activo en relación con los macrofactores del mercado, entonces podría eliminarse la volatilidad de los flujos de caja de ese activo que son sensibles ante las condiciones macroeconómicas o del mercado. En otras palabras, si pudiera cubrirse la primera variable que afecta el beta: la sensibilidad del activo ante el mercado, podría eliminarse o reducirse este componente como parte del cálculo del beta y disminuirse el riesgo sistemático del activo.

En un contrato de cobertura de las variables que reflejan la sensibilidad del activo ante 
condiciones del mercado, el precio y la demanda, podría reducirse o eliminarse el riesgo sistemático. Estas dos variables se ven reflejadas en la función de ventas de la firma. Por lo tanto, es posible llevar a cabo tanto coberturas separadas como coberturas conjuntas; es decir, podría llevarse a cabo una cobertura de precio o demanda, o una cobertura de ventas, que incluye tanto una cobertura de precio como una cobertura de la cantidad demandada del activo subyacente (gráficas 2, 3 y 4).

Gráfica 2

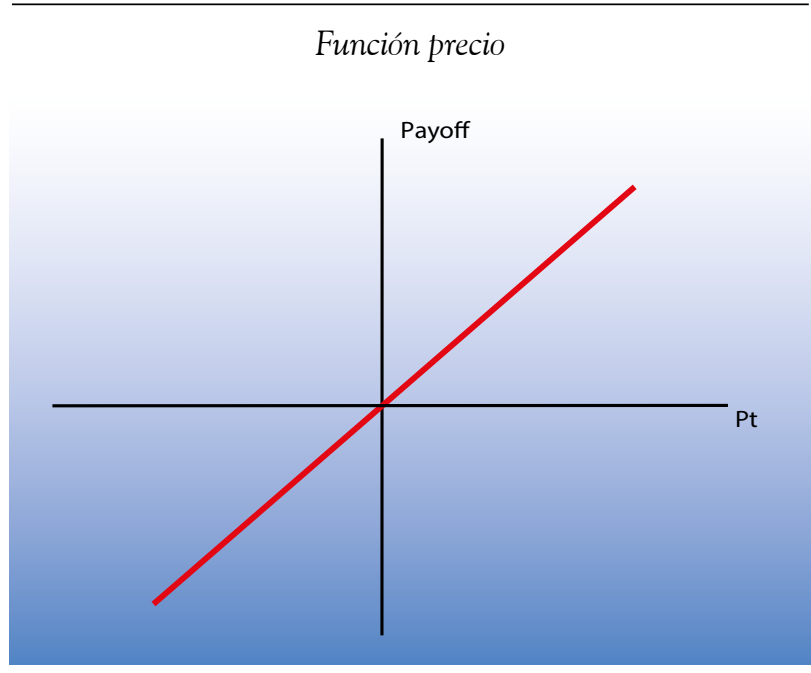

Fuente: elaboración de los autores.

\section{Gráfica 3}

Función demanda

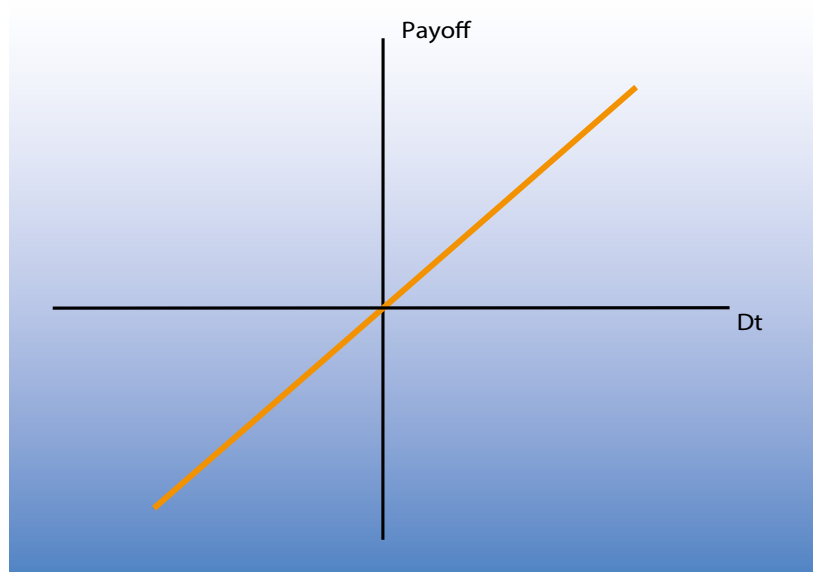

Fuente: elaboración de los autores.
Gráfica 4

Función ventas

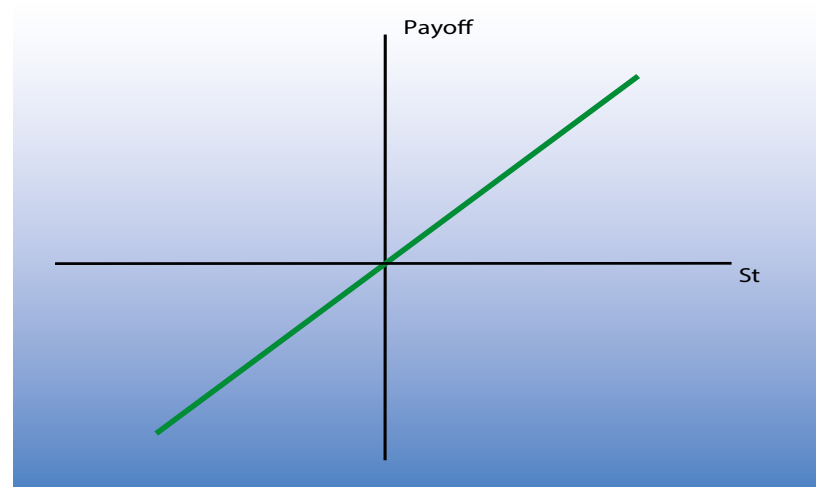

Fuente: elaboración de los autores.

Como puede apreciarse, las tres funciones tienen la misma exposición, en el sentido de que un incremento tanto en la demanda como en el precio, ceteris paribus, significará un beneficio para la empresa. El término beneficio no necesariamente significa lo mismo que utilidad del estado de pérdidas y ganancias, ya que la relación entre, por un lado, cambios de precio, cambios de demanda y cambios de ventas $y$, por otro, cambios de utilidad no es de 1:1, como aparece en las figuras anteriores; incluso, algunas empresas tienen una relación negativa entre las ventas y su utilidad: entre más venden, menos utilidad generan. Simplemente, el término beneficio es la diferencia de cambio sobre ese único activo como subyacente.

Dado que las tres funciones tienen las mismas exposiciones, pueden utilizarse instrumentos de una misma característica para aplicarse a todas. Sin embargo, al realizar una cobertura sobre ventas, se efectúa una cobertura tanto de precio como de demanda. A partir de lo anterior, procedemos entonces a la creación del derivado de ventas, teniendo en mente que lo que quiere lograrse es una maximización del valor del activo subyacente; para ello, tendremos en cuenta las dos variables que determinan un beta desapalancado $\left(\beta_{u}\right)$ : a) los factores de mercado que se reflejan a través del precio y de la demanda y que se manifiestan en la función de ventas y b) el 
grado de apalancamiento operativo de la empresa. Se harán las siguientes dos propuestas:

a) Al realizar una cobertura de ventas $y$, por ende, de los factores en los cuales se ve reflejada la sensibilidad del activo según condiciones macroeconómicas del mercado, es posible la disminución del beta desapalancado del activo, su riesgo sistemático y el aumento del valor de dicho activo.

b) El grado de apalancamiento operativo no se determina por la estructura de costos fijos y variables, sino por la función del EBIT (beneficio antes de intereses e impuestos). Lo anterior quiere decir que este se determina por la estructura operativa tanto de costos como de ingresos. Al realizar una cobertura de ventas que replique la estructura de costos, la volatilidad del EBIT disminuirá y, por ende, el beta desapalancado del activo, su riesgo sistemático y el aumento del valor de dicho activo.

Ahora bien, en primer lugar se requiere una definición del beta, a saber: la covarianza de los retornos del precio de un activo con los retornos del mercado:

$\beta_{i}=\frac{\sigma_{i, m}}{\sigma_{m}^{2}}$

Esto, claro está, puede aplicarse para empresas que cotizan en bolsa; para ellas, el único beta observable es el de las acciones (beta del equity). Sin embargo, para nuestro propósito, se supone que la empresa no tiene apalancamiento financiero: $\beta_{u}=\beta_{A}$ $=\beta_{E^{*}}$ Y aun cuando existiera apalancamiento financiero, es posible desapalancar el beta de las acciones o del patrimonio con los valores de mercado del patrimonio y de la deuda (observables en el mercado), para llegar así al beta desapalancado de los activos:

$\beta_{U}=\frac{(1-t) D}{(1-t) D+E} \beta_{D}+\frac{E}{(1-t) D+E} \beta_{E}$

Pero, ¿qué sucede cuando una empresa no cotiza en bolsa?, ¿qué ocurre cuando la empresa lleva poco tiempo cotizando y los datos no son lo suficientes para que la serie sea significativa? Una salida que proponen muchos teóricos es tomar el beta desapalancado ponderado de empresas que se encuentren en el mismo sector, ya que usualmente estas compañías tienen la misma tendencia a la sensibilidad ante factores macroeconómicos. Sin embargo, ¿qué sucede cuando una empresa no solo no cotiza en un mercado, sino que tampoco tiene empresas comparables? Una propuesta es utilizar el beta contable. Kulkarni, Powers y Shannon (1991) sugieren una técnica basada en el beta contable para calcular la tasa de descuento de una división en una compañía con productos múltiples. Como la información de mercado no estaba disponible, utilizaron el beta contable pare el cálculo de la tasa de descuento de esa división; así, concluyeron que este beta podría servir como una útil aproximación cuando el beta de mercado fuera difícil de calcular. La ecuación 10 refleja una aproximación matemática al beta contable y su cálculo:

$R_{i t}^{A}=\alpha+\beta_{i}^{A} R_{m t}^{A}+u_{i}$

En la anterior fórmula se halla el beta por medio de una regresión de los retornos de la empresa (utilidad operacional) y la regresión del mercado, lo cual también puede expresarse así:

$\beta_{A}=\frac{\operatorname{cov}\left(\% \Delta E B I T_{A}, \% \Delta E B I T_{m}\right)}{\operatorname{var}\left(\% \Delta E B I T_{m}\right)}$

Como puede apreciarse, los efectos del riesgo sistemático no se encuentran solo en la estructura de costos, sino más bien en la estructura del EBIT, que también incluye los efectos de los ingresos operacionales. Para lo anterior, se define el EBIT así:

$$
\begin{aligned}
E B I T_{t} & =S_{t}-C V_{t}-C F \\
& \text { Donde, } \\
& E B I T_{t}=\text { utilidad operacional antes de } \\
& \text { impuestos } \\
& C V_{t}=\text { costos variables operacionales en } \\
& \text { cualquier momento } t
\end{aligned}
$$


$C F=$ costos fijos operacionales

$C T_{t}=C V_{t}+C F=$ costos totales operacionales en cualquier momento $t$

Al igual que las ventas, los costos variables pueden representarse de la siguiente manera:

$C V_{t}=\sum_{i=1}^{n} C_{i t} Q_{i t}$

Donde,

$C_{i t}=$ costo unitario del producto $i$ en cualquier momento $t$ de tiempo

Se concluye que quiere suavizarse la variabilidad del EBIT para favorecer un menor riesgo sistemático. Al asumir una distribución normal en el comportamiento del precio, podrían modelarse las ventas a partir de la siguiente distribución (gráfica 5).

Gráfica 5

Ventas de un periodo 0 a un periodo $t$

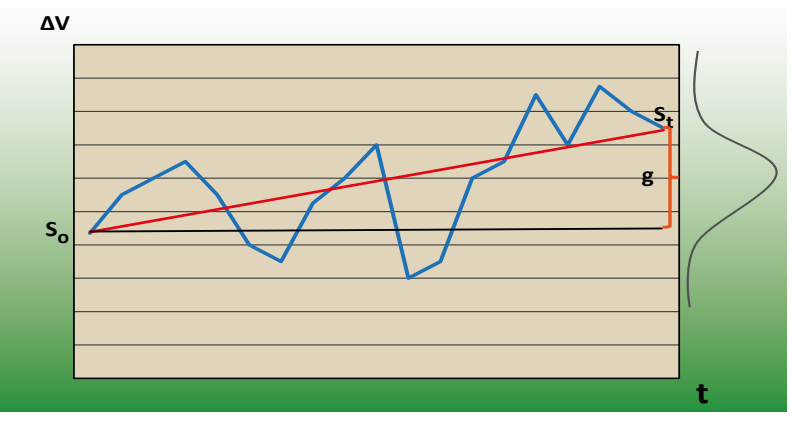

Fuente: elaboración de los autores.

Donde,

$S_{o}=$ las ventas hoy

$S_{t}=$ las ventas en el momento $t$

$g=$ crecimiento de las ventas

Allí las ventas pueden modelarse como un movimiento browniano simple:

$\partial S=\mu S \partial t+\sigma_{S} \partial z$

Se parte de la premisa de que las ventas son variables en su totalidad, y esta variabilidad está descrita por la función de demanda. Por otro lado, los costos tienen una estructura diferente: una parte fija y una parte variable. La diferencia entre las ventas y los costos totales (operacionales) en cualquier momento $t$ llevará a la utilidad o al beneficio marginal (gráfica 6).

Gráfica 6

Crecimiento de utilidad marginal

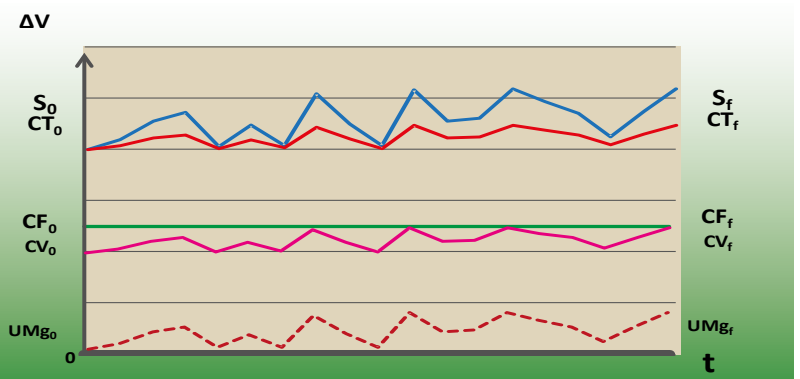

Fuente: elaboración de los autores.

Siempre y cuando se mantenga una tendencia de crecimiento, la curva de utilidad marginal será positiva y con mayores tendencias de crecimientos en las ventas, esto es, la curva de utilidad será marginalmente cada vez mayor. Sin embargo, cuando las ventas pasan por periodos de crecimientos negativos, la utilidad marginal comienza a decrecer marginalmente más rápido, y cuando el nivel de demanda cae por debajo del punto de equilibrio, la utilidad marginal será negativa. Esta curva de utilidad marginal es el EBIT, y los movimientos o las volatilidades en las curvas son el principal determinante en el beta contable. La gráfica 7 muestra lo que puede suceder cuando las ventas caen.

Gráfica 7

Decrecimiento de la utilidad marginal

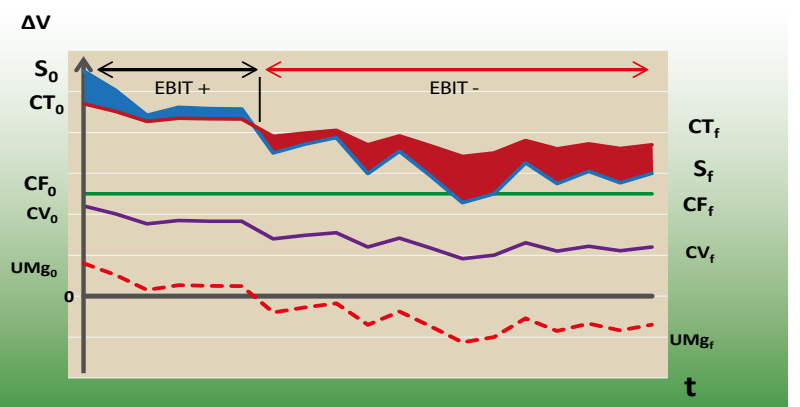

Fuente: elaboración de los autores. 
Según lo anterior, puede concluirse:

a) Los choques de la demanda ante efectos de mercado pueden ocasionar caídas en las ventas.

b) Estos choques pueden causar pérdidas en momentos en que la curva de función de ventas se encuentre por debajo de la curva de costos totales o incluso por debajo de la curva de costos fijos; es decir, las ventas o los ingresos pueden no ser suficientes para cubrir los costos totales o incluso, en algunas ocasiones, los costos fijos.

c) Estas altas variabilidades en la función de ventas con una estructura de costos fijos ocasionan una alta variabilidad en la función de utilidad marginal, o EBIT. Esta alta variabilidad del EBIT aumenta el riesgo sistemático de la compañía.

Para contrarrestar lo anterior, una opción es poder replicar la estructura de costos a partir de la estructura de ventas. El objetivo es poder minimizar la volatilidad del EBIT, lo cual puede lograrse a través de varias estrategias en la estructura de costos o en la de ventas o ingresos. Sin embargo, para este derivado en particular, el objetivo es simplemente realizar una cobertura de ventas; en otras palabras, ceteris paribus, cómo puede aumentarse el valor mediante una cobertura de ventas. Para ello, realizamos la estrategia mencionada: una replicación de la estructura de costos. La estructura de ventas puede descomponerse de la siguiente forma:

$S_{t}=\sum_{i=1}^{N} P_{i t} Q_{i t}$

A partir de ella, crearemos un derivado para ventas fijas, replicando la estructura de costos:

$S F=\frac{C F}{C F+C V_{0}} P_{0} Q_{0}$
También se replicará una estructura de ventas variables:

$S V_{t}=\frac{P_{t} Q_{t}}{S F+P_{t} Q_{t}} P_{t} Q_{t}$

Con estas dos funciones, puede modelarse la función de ventas así:

$S_{t}=S F+S V_{t}$

De acuerdo con este modelo, siempre y cuando en el momento $t=0$ se cumpla que $S_{t}>C T_{t^{\prime}}$ esta cobertura de réplica de la estructura de ventas resultará en que la empresa siempre generará un EBIT > 0 ; y adicionalmente las variaciones en el EBIT serán menores, ya que se habrá suavizado el efecto de choque entre la estructura de ingresos y la de costos. Como puede apreciarse en la gráfica 8, las ventas y los costos se descompondrían en un componente fijo y variable, proporcional a su estructura.

Gráfica 8

Ventas fijas y variables con crecimiento de utilidad marginal

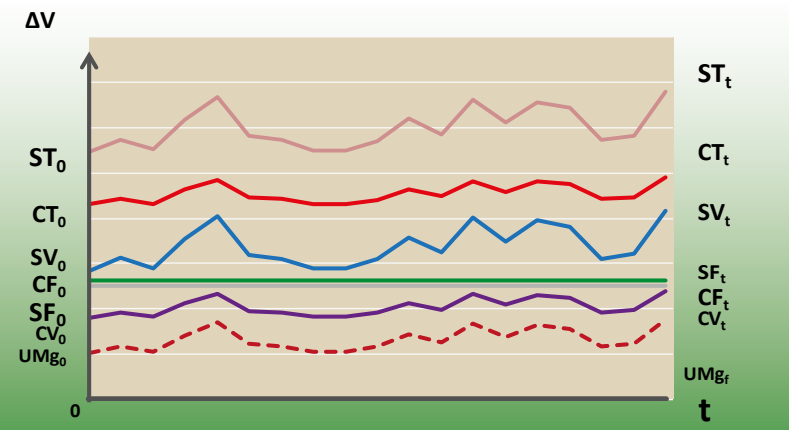

Fuente: elaboración de los autores.

No obstante, a diferencia de la anterior figura, e incluso en ciclos de caídas en las ventas, al replicar la estructura de ventas mediante una cobertura de ventas, a pesar de que habrá una leve caída en el EBIT, no se generarán pérdidas, y la varianza del EBIT se reducirá significativamente (compárense las gráficas 7 y 9).

Al observar el perfil de riesgo de las ventas en la gráfica 4, se concluye que diferentes instrumentos de cobertura pueden ser utilizados para cubrir el riesgo de caída de las ventas (downside risk). Entre 
Gráfica 9

Ventas fijas y variables con decrecimiento de utilidad marginal

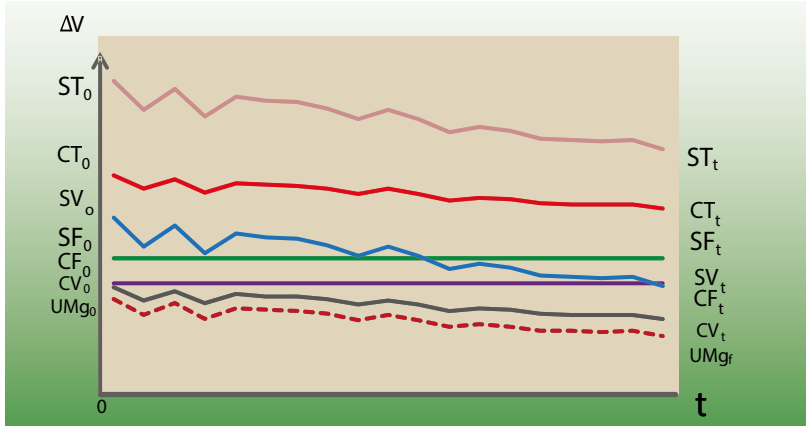

Fuente: elaboración de los autores.

estos instrumentos se encuentran los contratos forward, de futuros y de opciones, entre otros. Lo importante es entender que, más allá de que estos contratos pueden ser utilizados como instrumentos financieros, también son herramientas conceptuales factibles de armarse y desarmarse para formar la pieza o el instrumento que desean utilizarse. Teniendo en cuenta que no son más que derechos y obligaciones que se pactan en torno a uno o más activos subyacentes, Smithson (1987) compara a los derivados con figuras de $\mathrm{LEGO}^{\circledR}$, en función de ilustrar que para entender los derivados no se necesita saber demasiado acerca del mercado, sino comprender cómo unir las diferentes piezas.

Como se mencionó, pueden utilizarse diferentes instrumentos para llevar a cabo una cobertura de ventas. En su momento, lo importante para la estrategia no es pensar en cuántos instrumentos pueden utilizarse, sino en cuál de ellos es el más adecuado de acuerdo con su estructura. El contrato forward o de futuros es un intercambio de flujos en una fecha futura. Este contrato podría ser muy útil para llevar a cabo la cobertura de ventas, ya que es necesario un intercambio de flujos variables a flujos de fijos. Sin embargo, y dado que se requiere un intercambio de flujos en cada instante $\Delta t$, lo más adecuado sería un portafolio de forwards desde $t=0$ hasta $t=t$. Estos instrumentos son los que comúnmente se conocen como swaps; se hace un intercambio de una serie de flujos variables por una serie de flujos fijos. La gráfica 10 describe el comportamiento actual de las ventas; allí, el comportamiento es variable de un periodo a otro, resultado de los choques ocasionados por la distribución normal.

Gráfica 10

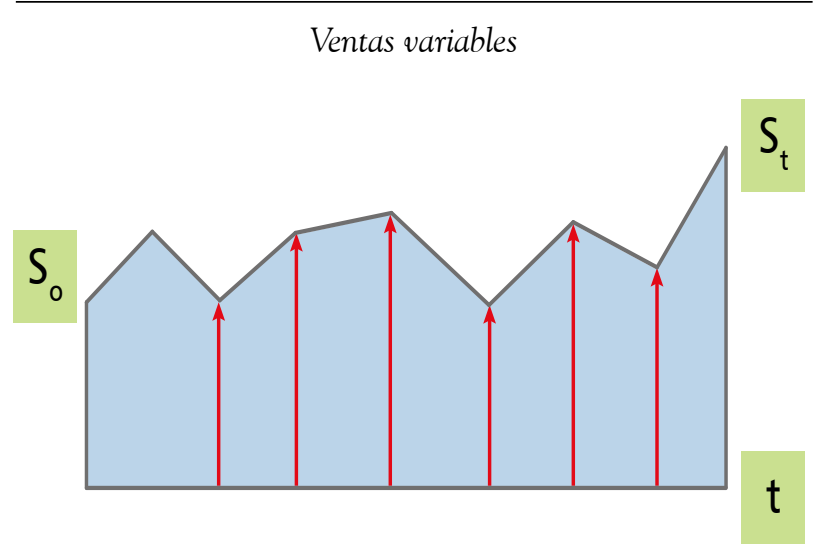

Fuente: elaboración de los autores.

A partir de lo anterior, se plantea el intercambio de un flujo variable por un flujo fijo en cada momento $t$, de forma que las ventas se vuelvan fijas (gráfica 11).

Gráfica 11

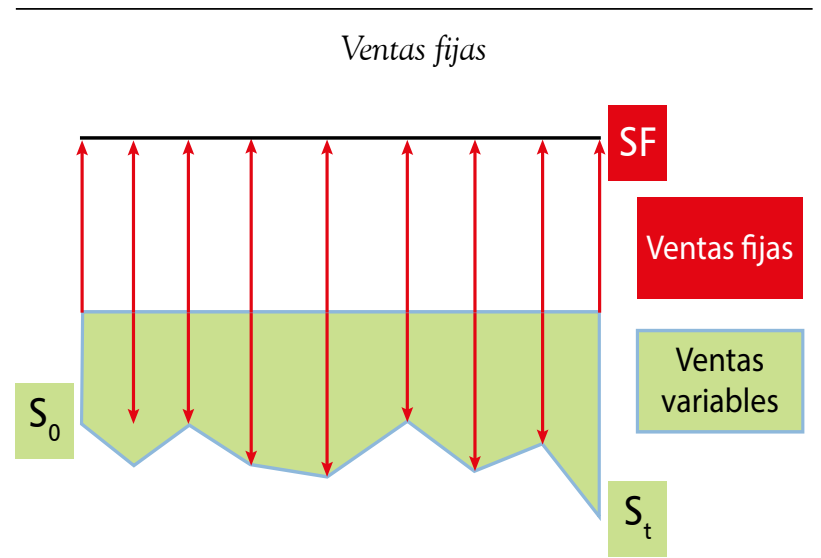

Fuente: elaboración de los autores.

El objetivo con este swap de ventas es poder cubrir en cada momento $t$ el riesgo al que están expuestas las ventas, dada la variabilidad de la demanda y el precio. Sin embargo, y a fin de crear valor para los accionistas, el objetivo final es disminuir la volatilidad del EBIT; para ello, si bien podría hacerse una cobertura total de las ventas (es decir, volver todas las ventas fijas), esto no 
resolvería el problema, sino que más bien cambiaría la variabilidad en el EBIT por causa de unos costos fijos a una variabilidad en el EBIT, resultado de unos costos variables. Lo que quiere lograrse, entonces, es una replicación de la estructura de costos, de forma que la estructura de costos fijos se encuentre cubierta por una estructura de ventas fijas, en tanto la estructura de costos variables sea cubierta por unas ventas variables. La gráfica 12 muestra cómo se cubriría el perfil de riesgo de la porción de ventas variables, que se convertirían en fijas a través de un instrumento financiero denominado swap sobre ventas.

\section{Gráfica 12}

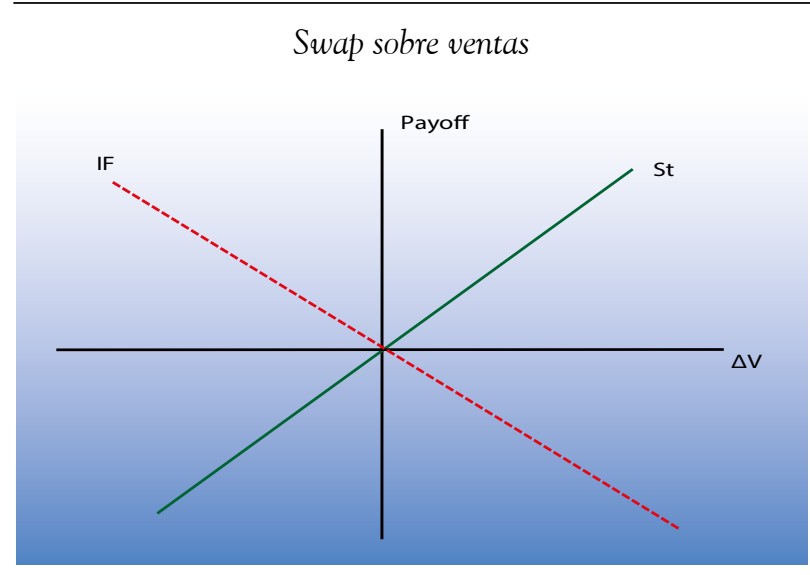

Fuente: elaboración de los autores.

Si bien se conoce el nivel de ventas en el momento 0 y la estructura de crecimiento, lo que quiere saberse es cuánto sería el valor de las ventas fijas a través del cual se llevaría a cabo el swap. Para ello, se plantea el siguiente modelo matemático:

$S_{t}=S_{t-\Delta t} e^{g \Delta t}+\varepsilon_{t} \rightarrow \varepsilon_{t} \sim N(0, \sigma) I I D$

Cuando $\Delta t=t$, la ecuación se simplifica así:

$S_{t}=S_{0} e^{g t}+\varepsilon_{t} \rightarrow \varepsilon_{t} \sim N(0, \sigma) I I D$

El valor esperado de la función $S_{t}$ puede resumirse en las siguientes dos ecuaciones:

$E\left[S_{t}\right]=E\left[S_{0} e^{g t}\right]+E\left[\varepsilon_{t}\right]$
$E\left[S_{t}\right]=S_{0} e^{g t}$

A partir de lo anterior, definiremos el valor del swap sin tener en cuenta su efecto sobre el valor de la empresa:

$V($ swap $)=V($ ventas variables $)-V($ ventas fijas $)=0$

$V($ ventas variables $)=V($ ventas fijas $)$

Ahora puede expresarse en términos continuos la ecuación anterior:

$V($ ventas variables $)=\int_{0}^{t} \frac{S_{0} e^{g i}}{e^{r i}} \partial i$

$V($ ventas fijas $)=\int_{0}^{t} \frac{S F}{e^{r i}} \partial i$

Donde,

$g=$ crecimiento continuo del periodo $\partial i$ de capitalización

$r=$ tasa libre de riesgo

Las demás variables ya han sido definidas. Así, al igualar las dos ecuaciones, se tiene:

$$
\begin{aligned}
& \int_{0}^{t} \frac{S_{0} e^{g i}}{e^{r i}} \partial i=\int_{0}^{t} \frac{S F}{e^{r i}} \partial i \\
& S_{0} \int_{0}^{t} e^{(g-r) i} \partial i=S F \int_{0}^{t} e^{-r i} \partial i \\
& \frac{S_{0}}{g-r}\left[e^{(g-r) i}\right]_{0}^{t}=\frac{S F}{-r}\left[e^{-r i}\right]_{0}^{t}
\end{aligned}
$$

Con esto se llega a la solución que se expresa en esta ecuación:

$S F^{*}=\left[\frac{\left(S_{0}-S_{0} e^{(g-r) t}\right) r}{(r-g)\left(1-e^{-r t}\right)}\right]$ 
La ecuación 27 también puede expresarse en términos discretos así:

$$
S F^{*}=S_{0} \frac{\sum_{i=1}^{t} \frac{(1+g)^{i}}{(1+r)^{i}}}{\sum_{i=1}^{t} \frac{1}{(1+r)^{i}}}
$$

Para comprobar los anteriores resultados, se llevó a cabo una simulación de las ventas tanto en tiempo discreto como en tiempo continuo, para lo cual se tomó una muestra suficientemente grande. Los que siguen fueron los resultados de la simulación (tablas 1-3).
Tabla 1

\begin{tabular}{|c|c|}
\hline \multicolumn{2}{c}{ Datos de entrada } \\
\hline SO & 80.000 \\
\hline R & $10 \%$ \\
\hline G & $3 \%$ \\
\hline T & 2996 \\
\hline
\end{tabular}

Fuente: elaboración de los autores.

Por efectos de espacio, se muestran solo los primeros treinta datos, simulados desde $t=0$ hasta $\mathrm{t}=2996$.

Simulación del swap de ventas

\begin{tabular}{|c|c|c|c|}
\hline$t$ & Ventas variables-discretas & Ventas variables-continuas & Ventas fijas \\
\hline 0 & 80 & 80 & 114.286 \\
\hline 1 & 82.400 & 82.436 & 114.286 \\
\hline 2 & 84.872 & 84.947 & 114.286 \\
\hline 3 & 87.418 & 87.534 & 114.286 \\
\hline 4 & 90.041 & 90.200 & 114.286 \\
\hline 5 & 92.742 & 92.947 & 114.286 \\
\hline 6 & 95.524 & 95.777 & 114.286 \\
\hline 7 & 98.390 & 98.694 & 114.286 \\
\hline 8 & 101.342 & 101.700 & 114.286 \\
\hline 9 & 104.382 & 104.797 & 114.286 \\
\hline 10 & 107.513 & 107.989 & 114.286 \\
\hline 11 & 110.739 & 111.277 & 114.286 \\
\hline 12 & 114.061 & 114.666 & 114.286 \\
\hline 13 & 117.483 & 118.158 & 114.286 \\
\hline 14 & 121.007 & 121.757 & 114.286 \\
\hline 15 & 124.637 & 125.465 & 114.286 \\
\hline 16 & 128.377 & 129.286 & 114.286 \\
\hline 17 & 132.228 & 133.223 & 114.286 \\
\hline 18 & 136.195 & 137.281 & 114.286 \\
\hline 19 & 140.280 & 141.461 & 114.286 \\
\hline 20 & 144.489 & 145.770 & 114.286 \\
\hline 21 & 148.824 & 150.209 & 114.286 \\
\hline 22 & 153.288 & 154.783 & 114.286 \\
\hline 23 & 157.887 & 159.497 & 114.286 \\
\hline 24 & 162.624 & 164.355 & 114.286 \\
\hline 25 & 167.502 & 169,36 & 114.286 \\
\hline 26 & 172.527 & 174.518 & 114.286 \\
\hline 27 & 177.703 & 179.833 & 114.286 \\
\hline 28 & 183.034 & 185.309 & 114.286 \\
\hline 29 & 188.525 & 190.953 & 114.286 \\
\hline 30 & 194.181 & 196.768 & 114.286 \\
\hline
\end{tabular}

Fuente: elaboración de los autores. 
Tabla 3

Resultados del swap de ventas

\begin{tabular}{|c|c|}
\hline Modelo & Resultado \\
\hline SF & 114.286 \\
\hline Ventas fijas & 1.142 .857 \\
\hline Ventas variables & 1.142 .857 \\
\hline Valor swap & 0,00 \\
\hline
\end{tabular}

Fuente: elaboración de los autores.

Como puede apreciarse, el valor presente de las ventas fijas es igual al valor presente de las ventas variables; por ende, el valor de intercambio del swap es 0 . Sin embargo, el objetivo de este es la creación de valor para la empresa, lo que puede conocerse como el valor generado por el swap de ventas (diferente al valor de intercambio del swap de ventas, que siempre debe de ser 0 ). Para lograr lo anterior, no es necesario hacer un swap sobre la totalidad de las ventas, sino sobre la proporción que quiere replicarse en los costos fijos. Para ello, esta simplemente se sustituye en la ecuación 27 y 28 por la proporción de ventas que replique la estructura de costos fijos. Esta proporción se encuentra planteada en la ecuación 16, donde se había determinado la estructura a partir de la cual se crearía el derivado de ventas.

En las ecuaciones 27 y 28 se sustituye So por $S_{0}^{*}$, de lo que resulta:

$S_{0}^{*}=\frac{C F}{C F+C V_{0}} S_{0}$

A partir de la anterior replicación de la estructura de costos fijos, el swap quedaría definido así:

$S F^{*}=\left[\frac{\left.S_{0}^{*}-S_{0}^{*} e^{(g-r) t}\right) r}{(r-g)\left(1-e^{-r t}\right)}\right]$

$S F^{*}=S_{0}^{*} \frac{\sum_{i=1}^{t} \frac{(1+g)^{i}}{(1+r)^{i}}}{\sum_{i=1}^{t} \frac{1}{(1+r)^{i}}}$
Con la anterior estructura se habría creado un swap de ventas que no solo convierte las ventas variables en ventas fijas, sino que adicionalmente replica la estructura de costos fijos y variables. Más adelante se medirá el efecto de los derivados de pérdidas y ganancias sobre el valor de una empresa o un activo. Sin embargo, por lo pronto se consideran las siguientes conclusiones respecto a una cobertura de ventas:

a) Permite que una empresa siempre tenga un EBIT o una utilidad marginal mayor a 0 , siempre y cuando en el momento $t=$ 0 el EBIT sea mayor a 0 .

b) Disminuye la sensibilidad de la empresa ante factores macroeconómicos, ya que la función de precios y demanda determina en gran parte la sensibilidad ante factores macroeconómicos. Al realizar una cobertura de ventas, se disminuye el efecto de estos factores.

c) El segundo punto debería llevar a una disminución del beta desapalancado de la empresa o el activo.

d) La réplica de la estructura de costos disminuye las variaciones del EBIT, lo cual lleva a una disminución en el beta desapalancado de la empresa.

e) Si bien existen intercambios de flujos variables por fijos, el valor esperado de los flujos no cambia; es decir, los flujos simplemente se modifican de naturaleza variable a fija.

f) Al no verse modificados los flujos de caja, y al contar con un menor riesgo sistemático (beta), la empresa o el activo tendrán un mayor valor.

\section{DERIVADO REAL DE COSTOS}

Según Kaplan, respecto a la estructura de costos, "la habilidad de una compañía para innovar, mejorar y aprender está directamente relacionada con el valor de esa compañía". Los costos pueden describirse como un recurso que se sacrifica para 
alcanzar un objetivo específico, a saber: la generación de ingresos y, más allá, la creación de valor para los accionistas. Al seguir con el lineamiento de este trabajo, puede apreciarse que el objetivo final de cualquier recurso que se invierta en costo será la generación o creación de valor. Para poder generar valor a los accionistas, en ambientes competitivos, las empresas tendrán que recurrir cada vez más a las herramientas que están a su disposición, o bien, a aquellas que puedan desarrollar.

En este contexto, la estructura de costos desempeña un papel importante. Esta puede desagregarse en costos fijos y costos variables: los primeros son aquellos que no cambian a pesar de las variaciones producidas en los factores de demanda, los segundos son los que cambian de acuerdo con las variaciones producidas en los factores de demanda. Si bien algunos costos pueden considerarse como semivariables, en su naturaleza estos pueden desagregarse en sus partes variable y fija. La estructura de costos puede expresarse de la siguiente manera:

$$
\begin{aligned}
& C V_{t}=\sum_{i=1}^{n} C_{i t} Q_{i t} \\
& C F=\bar{K} \\
& C T=C V_{t}+C F
\end{aligned}
$$

Donde, $C V_{t}=$ costos variables operacionales en cualquier momento $t$

$C_{i t}=$ costo unitario del producto $i$ en cualquier momento $t$ de tiempo

$C F=$ costos fijos operacionales

$C T_{t}=$ costos totales operacionales en cualquier momento $t$

La estructura de costos fijos y costos variables muchas veces depende del tipo de negocio. Existen sectores de la economía que, por su tipo de operación, tienden a disponer cierta estructura de costos. Sin embargo, en muchas ocasiones, la estructura de costos fijos y variables son decisiones de gerencia: los costos son fijos porque las gerencias los conceptualizaron de esa manera, es decir, hicieron contratos para convertirlos en tales. No obstante, surge la pregunta: ¿cuál es el problema de tener costos fijos? Según Damodaran (2002), todo lo demás sigue siendo igual: empresas que tengan una mayor proporción de costos fijos tendrán también un mayor riesgo sistemático. Pero el problema no subyace solo en la estructura de costos, sino en la estructura de EBIT, la cual es base para las proyecciones de los flujos de caja libre por medio de la cual la empresa será valorada.

La afirmación de que entre mayor sea la proporción de costos fijos que una empresa tenga, mayor será su riesgo sistemático depende de un supuesto: las ventas son variables. Pero como se demostró en la sección anterior, es posible crear un instrumento que convierta el componente variable de dichas ventas en un componente fijo. Según esto, no podría afirmarse que necesariamente una mayor estructura de costos fijos aumentaría el riesgo sistemático de la empresa; lo que sí puede asegurarse es que una menor variabilidad en el EBIT sí afectaría el riesgo sistemático de la empresa. En consecuencia, una menor variabilidad en el EBIT aumentaría el valor de la empresa. Lo importante es tener en cuenta que, en su naturaleza, las ventas son variables y, por ende, lo lógico sería poder llevar a cabo una cobertura de costos fijos, a fin de disminuir la variabilidad en el EBIT. El perfil de riesgo de la estructura de costos se puede apreciar en las siguientes gráficas.

Gráfica 13

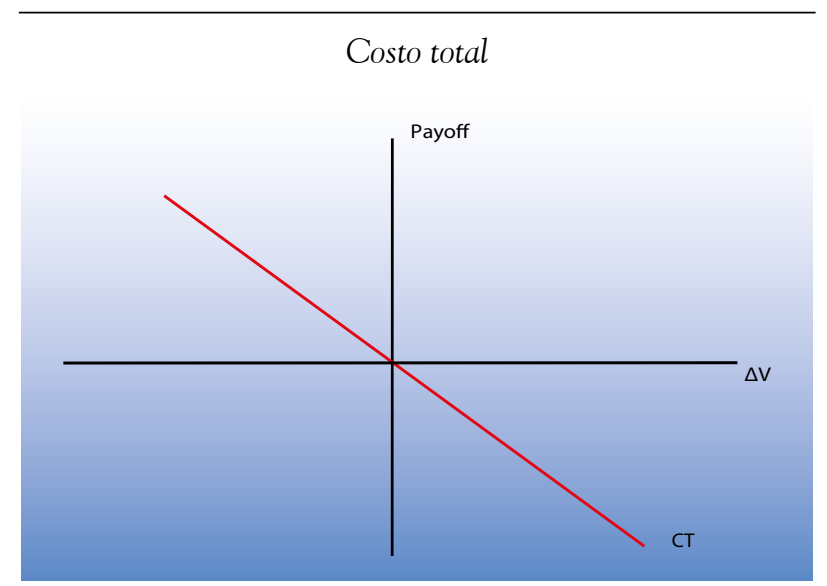

Fuente: elaboración de los autores. 


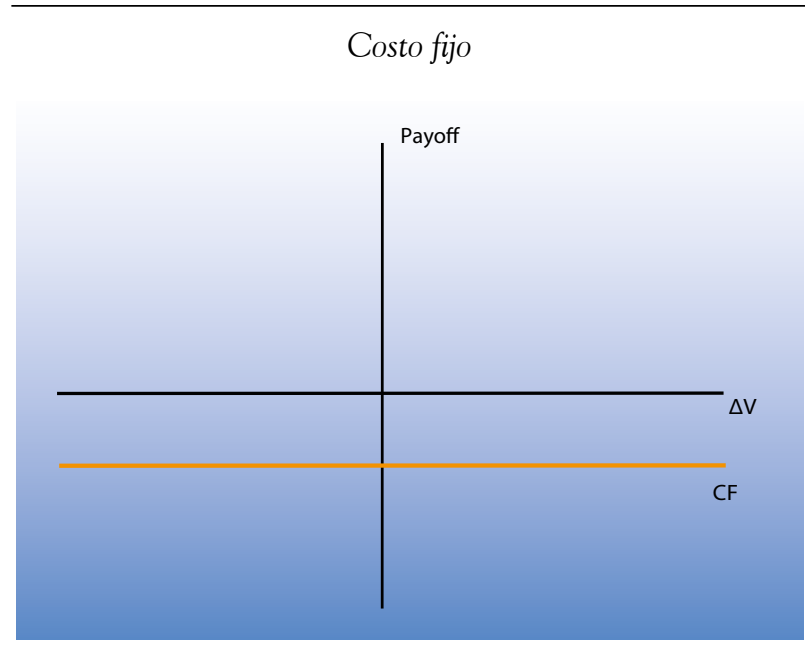

Fuente: elaboración de los autores.

\section{Gráfica 15}

Costo variable

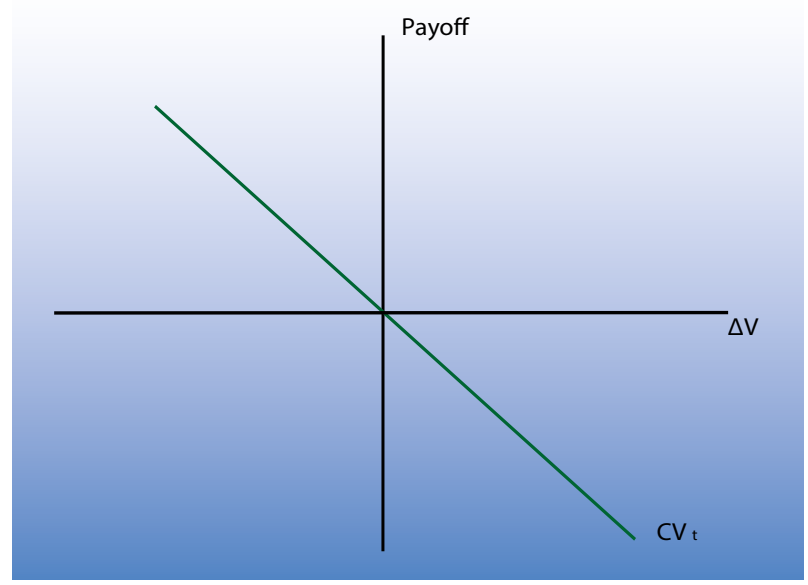

Fuente: elaboración de los autores.

Al igual que con los derivados de ventas, lo que quiere lograrse en los derivados de costos es el intercambio de flujos fijos a variables, y también, en ocasiones, de flujos variables a fijos. Por ello, el instrumento indicado sería un swap de flujos de costos. Las gráficas 16 y 17 muestran cómo se implementaría un swap de costos fijos y de costos variables para el intercambio de esta exposición al riesgo.
Gráfica 16

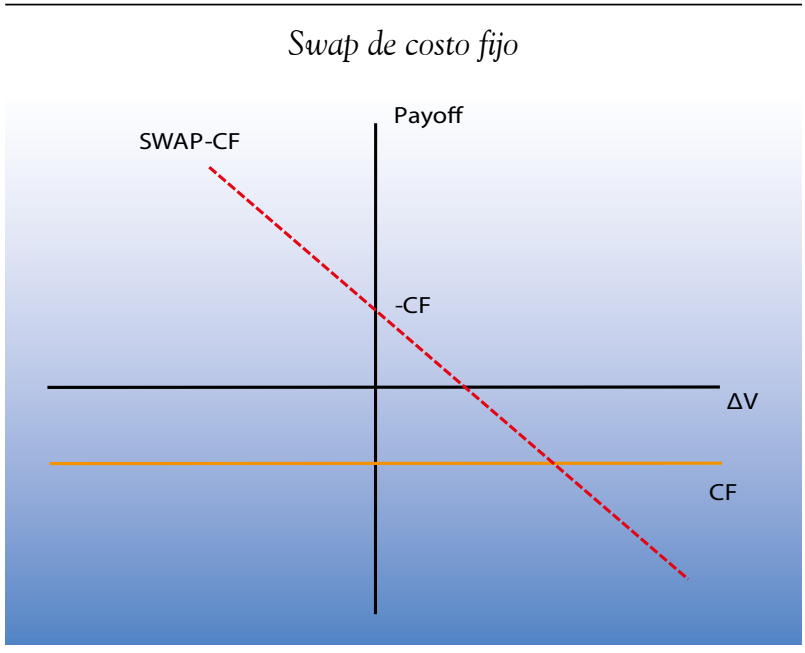

Fuente: elaboración de los autores.

Gráfica 17

Swap de costo variable

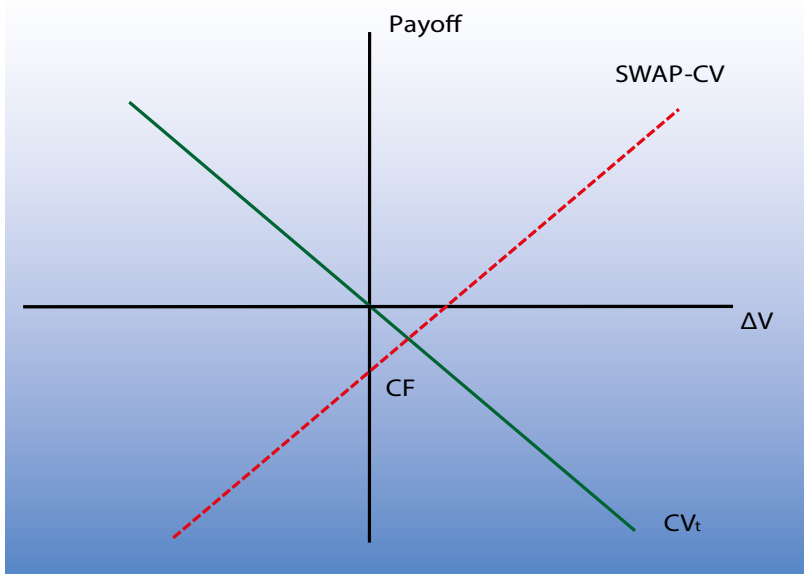

Fuente: elaboración de los autores.

El swap de costos debería poder plantearse de manera similar al de ventas. Según las hipótesis de Fama (1970), en un mercado eficiente, los precios pueden modelarse como una caminata aleatoria o un movimiento browniano simple, también conocidos como proceso estocástico Wiener. En términos reales, la función del precio puede expresarse de la siguiente manera:

$$
\begin{aligned}
& P_{t}=P_{t-1}+\varepsilon_{t} \rightarrow \varepsilon_{t} \sim N(0, \sigma), I I D \\
& E\left(P_{t}\right)=E\left(P_{t-1}\right)+E\left(\varepsilon_{t}\right)=E\left(P_{t-1}\right)=P_{0}
\end{aligned}
$$


Lo anterior es consistente con las teorías de eficiencia del mercado, según las cuales el precio de hoy es el mejor estimativo para el precio futuro de un activo. Sin embargo, claramente puede observarse que la función de ventas presenta un crecimiento; además, dado que esta función depende tan solo de dos variables, si ese crecimiento no es atribuible a uno en el precio, entonces debe ser un crecimiento en la demanda. Como ese crecimiento se presenta en las cantidades demandadas de los bienes $Q^{*}$, entonces también debe presentarse el mismo crecimiento en la estructura de costos variables. Lo anterior es consistente por cuanto el comportamiento de las ventas y de los costos variables es igual. De ahí que sea común utilizar el "margen de contribución", que elimina las cantidades y se halla de la siguiente forma desde el supuesto de que no hay economías de escala:

Margen de contribución $=\frac{S_{t}-C V_{t}}{Q_{t}}=\frac{\left(P_{t}-C_{t}\right) Q_{t}}{Q_{t}}=P_{t}-C_{t}$

Lo anterior quiere decir que los costos variables pueden formularse así:

$C V_{t}=C V_{t-\Delta t} e^{g \Delta t}+\varepsilon_{t} \rightarrow \varepsilon_{t} \sim N(0, \sigma) I I D$

Cuando $\Delta t=t$, la ecuación se simplifica así:

$C V_{t}=C V_{0} e^{g t}+\varepsilon_{t} \rightarrow \varepsilon_{t} \sim N(0, \sigma) I I D$

El valor esperado de la función $C V_{r}$ puede resumirse en las siguientes dos ecuaciones:

$E\left[C V_{t}\right]=E\left[C V_{0} e^{g t}\right]+E\left[\varepsilon_{t}\right]$

$E\left[C V_{t}\right]=C V_{0} e^{g t}$ fijos es:

Mientras tanto, la estructura de los costos

$C F=\bar{K}$

Por ello, para hacer el swap se requiere una tasa de conversión que tenga en cuenta la anterior estructura, recordando que $V($ swap $)-0 \mathrm{y}$, por tanto, $V($ costos fijos $)-V($ costos variables). En primer lugar se plantea el swap de costos fijos:

$$
\begin{aligned}
& V(\text { costos variables })=\int_{o}^{t} \frac{C V_{0} e^{g i}}{e^{r i}} \partial i \\
& V(\text { costos fijos })=\int_{0}^{t} \frac{C F}{e^{r i}} \partial i
\end{aligned}
$$

Al igualar y resolver de la misma forma como se hizo en la ecuación 27 , se obtiene:

$C F^{-}=\left[\frac{\left(C V_{0}-C V_{0} e^{(g-r) t}\right) r}{(r-g)\left(1-e^{-r t}\right)}\right]$

Esta también puede expresarse en términos discretos así:

$C F^{\rightarrow}=C V_{0} \frac{\sum_{i=1}^{t} \frac{(1+g)^{i}}{(1+r)^{i}}}{\sum_{i=1}^{t} \frac{1}{(1+r)^{i}}}$

Para hacer el swap de costo fijos, se resuelve la misma ecuación por $C V_{0}$, teniendo en cuenta que puede sustituirse el valor de $C V_{0}$ en la ecuación 39. De esta forma, se llega a las siguientes ecuaciones:

$C V_{0}^{*}=\frac{\left(C F-C F e^{-r t}\right)(r-g)}{r\left(1-e^{(g-r) t}\right)}$

Esta también puede expresarse en términos discretos así:

$C V_{0}^{*}=C F \frac{\sum_{i=1}^{t} \frac{1}{(1+r)^{i}}}{\sum_{i=1}^{t} \frac{(1+g)^{i}}{(1+r)^{i}}}$

A partir de lo anterior, y sustituyendo en la ecuación 39, se obtiene:

$C V_{t}^{*}=\frac{\left(C F-C F e^{-r t}\right)(r-g)}{r\left(1-e^{(g-r) t}\right)} e^{g t}+\varepsilon_{t} \rightarrow \varepsilon_{t} \sim N(0, \sigma) I I D$ 
La cual también puede expresarse en términos discretos como:

$$
C V_{t}^{*}=C F \frac{\sum_{i=1}^{t} \frac{1}{(1+r)^{i}}}{\sum_{i=1}^{t} \frac{(1+g)^{i}}{(1+r)^{i}}} e^{g t}+\varepsilon_{t} \rightarrow \varepsilon_{t} \sim N(0, \sigma) I I D
$$

Luego de haber realizado la cobertura de costos fijos (o variables), es importante medir el impacto que esta tiene sobre el beta desapalancado de un activo, en aras de poder determinar el impacto de una cobertura de costos sobre el valor del activo. Para ello, el grado de apalancamiento es una de las tres variables que determina el riesgo sistemático (beta). Según Besley y Brigham (2001), el grado de apalancamiento operativo puede definirse como el cambio porcentual en el EBIT ante un cambio porcentual en las ventas y puede formularse así:

$G A O=\frac{\% \Delta E B I T}{\% \Delta V E N T A S}=\frac{\frac{E B I T_{t}-E B I T_{t-1}}{E B I T_{t-1}}}{\frac{S_{t}-S_{t-1}}{S_{t-1}}}$

Ahora se sustituye en función las variables ya representadas, para poder mirar el impacto que tiene la estructura de costos sobre el grado de apalancamiento operativo de una empresa y sobre el beta. Primero se hace sustitución:

$$
\begin{aligned}
& E B I T_{t}=S_{t}-C V_{t}-C F \\
& \% \Delta E B I T=\frac{\left[S_{t}-C V_{t}-C F\right]-\left[S_{t-1}-C V_{t-1}-C F\right]}{\left[S_{t-1}-C V_{t-1}-C F\right]} \\
& \% \Delta E B I T=\frac{\left[S_{t}-C V_{t}\right]-\left[S_{t-1}-C V_{t-1}\right]}{\left[S_{t-1}-C V_{t-1}-C F\right]} \\
& \% \triangle E B I T=\frac{\left[Q_{t}-Q_{t-1}\right][P-C]}{\left[Q_{t-1}(P-C)-C F\right]}
\end{aligned}
$$

Al introducir la ecuación 37 en la 49, se obtiene:

$G A O=\frac{\% \Delta E B I T}{\% \Delta V E N T A S}=\frac{\frac{\left[Q_{t}-Q_{t-1}\right][P-C]}{\left[Q_{t-1}(P-C)-C F\right]}}{\frac{Q_{t}-Q_{t-1}}{Q_{t-1}}}$

A partir de la anterior ecuación puede verse que si $C F=0$, es decir, que todos los costos son variables, entonces el grado de apalancamiento operativo es igual a 1. No necesariamente todos los costos tendrían que ser variables desde un comienzo, pues estos pudieron haber sido fijos y después, a través de un swap, ser convertidos en variables. En la siguiente ecuación se define el beta contable:

$\beta_{A}=\frac{\operatorname{cov}\left(\% \Delta E B I T_{A}, \% \Delta E B I T_{m}\right)}{\operatorname{var}\left(\% \Delta E B I T_{m}\right)}$

A partir de lo anterior, la covarianza puede matematizarse así:

$\operatorname{cov}(x, y)=\frac{1}{n} \sum_{i=1}^{n}\left(x_{i}-\bar{x}\right)\left(y_{i}-\bar{y}\right)$

Por lo tanto, se obtiene:

$E B I T_{i}=S_{i}-C V_{i}-C F$

$\% \Delta E B I T=\frac{E B I T_{i}^{A}-E B I T_{i-1}^{A}}{E B I T_{i-1}^{A}}=\frac{\left[S_{i}-C V_{i}\right]-\left[S_{i-1}-C V_{i-1}\right]}{\left[S_{i-1}-C V_{i-1}-C F\right]}=\frac{\left[Q_{i}-Q_{i-1}\right][P-C]}{\left[Q_{i-1}(P-C)-C F\right]}$

De manera específica, puede valorarse ya la estrategia del swap de costos fijos, cuyo propósito es intercambiarlos por unos costos variables, de forma que la estructura de costos pueda replicar la variabilidad de las ventas y disminuir la volatilidad del EBIT. En este caso, los costos fijos desaparecen en su totalidad $(C F=0)$, y toda la estructura de costos se convierte en variable. 
Según esto, la ecuación 57 puede expresarse de la siguiente manera con swap de costos fijos:

$\% \Delta E B I T=\frac{E B I T_{i}^{A}-E B I T_{i-1}^{A}}{E B I T_{i-1}^{A}}=\frac{\left[S_{i}-C V_{i}\right]-\left[S_{i-1}-C V_{i-1}\right]}{\left[S_{i-1}-C V_{i-1}\right]}=\frac{\left[Q_{i}-Q_{i-1}\right]}{\left[Q_{i-1}\right]}[58]$

Debe recordarse que $g$ representa el crecimiento esperado de la demanda en espacio de tiempo $\Delta l$ :

$E[\% \Delta E B I T]=E\left[\frac{E B I T_{i}^{A}-E B I T_{i-1}^{A}}{E B I T_{i-1}^{A}}\right]=E\left[\frac{\left[S_{i}-C V_{i}\right]-\left[S_{i-1}-C V_{i-1}\right]}{\left[S_{i-1}-C V_{i-1}\right]}\right]=E\left[\frac{\left[Q_{i}-Q_{i-1}\right]}{\left[Q_{i-1}\right]}\right]=g[59]$

Al sustituir la ecuación 59 en la 55 , se obtiene:

$\operatorname{cov}\left(\% \Delta E B I T_{A^{\prime}} \% \Delta E B I T_{m}\right)=\frac{1}{n} \sum_{i=1}^{n}\left(\frac{\left[Q_{i}-Q_{i-1}\right]}{\left[Q_{i-1}\right]}-g\right)\left(\frac{E B I T_{i}^{M}-E B I T_{i-1}^{M}}{E B I T_{i-1}^{M}}-E\left[\frac{E B I T_{i}^{M}-E B I T_{i-1}^{M}}{E B I T_{i-1}^{M}}\right)\right)$

Como puede apreciarse, el beta pasa a estar determinado por cambios no esperados en el crecimiento de la demanda con respecto al mercado, lo cual representa una disminución significante en el beta del activo. Lo anterior puede comprobarse ya que los cambios en el EBIT se manifiestan a través de dos variables: cambios no esperados en la demanda (también puede representarse como la sensibilidad de la cantidad demandada de la empresa ante factores macroeconómicos) y el grado de apalancamiento operativo:

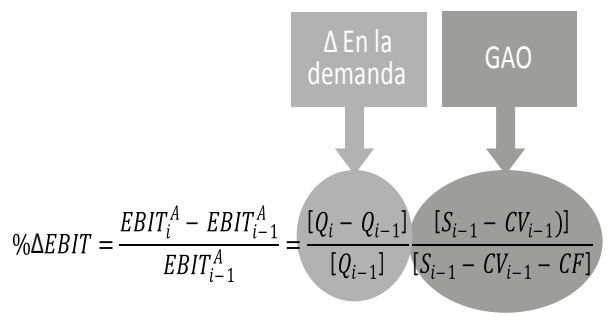

Esto quiere decir que el beta de un activo sin palanca financiera está determinado por dos factores: los cambios no esperados en la demanda y el grado de apalancamiento operativo. Es obvio que mientras los costos fijos no puedan tomar valores negativos, y mientras la empresa no parta de una estructura de pérdidas, tanto una empresa como un activo jamás podrán tener un grado de apalancamiento operativo menor a 1, lo cual puede comprobarse así:
$\mathrm{Si}$,

$S_{t-1} \geq 0$

$C V_{\mathrm{t}-1} \geq 0$

$C F \geq 0$

$S_{t-1}-C V_{t-1}-C F \geq 0$

Comprobación:

$C F \geq 0$ lados:

Luego se suma $C V_{t-1}$ y se resta $S_{t-1}$ en ambos $C F+C V_{t-1}-S_{t-1} \geq 0+C V_{t-1}-S_{t-1}$

Después, ambos se multiplican por -1 y se reorganizan:

$S_{t-1}-C V_{t-1}-C F \leq S_{t-1}-C V_{t-1}$

Al dividir ambos lados por $S_{t-1}-C V_{t-1}-C F$, se obtiene:

$\frac{S_{t-1}-C V_{t-1}-C F}{S_{t-1}-C V_{t-1}-C F} \leq \frac{S_{t-1}-C V_{t-1}}{S_{t-1}-C V_{t-1}-C F}$

Al reorganizar, finalmente se obtiene esta ecuación:

$\frac{S_{t-1}-C V_{t-1}}{S_{t-1}-C V_{t-1}-C F} \geq 1$

Se concluye entonces que, en tanto se asuman ventas variables, eliminar los costos fijos suprime también el componente de grado de apalancamiento del beta. Ahora bien, para mirar el impacto que tiene un swap de costos fijos sobre el beta de una empresa o un activo, se halla el $\Delta$ beta del activo, definido como la diferencia entre el beta desapalancado del activo antes y después de hacer la cobertura:

$\Delta \beta_{U}^{A}=\beta_{U}^{U}-\beta_{U}^{H}$ 
Donde,

$\Delta \beta_{U}^{A}=$ diferencia entre el beta sin cobertura y el beta con cobertura

$\beta_{U}^{U}=$ beta sin cobertura (unhedged)

$\beta_{U}^{H}=$ beta con cobertura (hedged)

$\beta_{U}^{U}=\frac{\sum_{i=1}^{n}\left(\left[\frac{\left[Q_{i}-Q_{i-1}\right]}{\left[Q_{i-1}\right]}-g\right]\right)\left(\% \Delta E B I T_{i}^{M}-E\left[\% \Delta E B I T_{i}^{M}\right]\right)}{\operatorname{var}\left(\% \Delta E B I T_{i}^{M}\right)} G A 0$

$\beta_{U}^{H}=\frac{\sum_{i=1}^{n}\left(\left[\frac{\left[Q_{i}-Q_{i-1}\right]}{\left[Q_{i-1}\right]}-g\right]\right)\left(\% \Delta E B I T_{i}^{M}-E\left[\% \Delta E B I T_{i}^{M}\right]\right)}{\operatorname{var}\left(\% \Delta E B I T_{i}^{M}\right)}$

$\Delta \beta_{U}^{A}=\frac{\sum_{i=1}^{n}\left(\left[\frac{\left.Q_{i}-Q_{i-1}\right]}{\left[Q_{i-1}\right]}-g\right]\right)\left(\% \Delta E B I T_{i}^{M}-E\left[\% \Delta E B I T_{i}^{M}\right]\right)}{\operatorname{var}\left(\% \Delta E B I T_{i}^{M}\right)(G A O-1)}$

De esta forma, el valor generado por el swap de costos fijos es el siguiente:

$\Delta$ Valor activo $=$ valor activo ${ }^{H}-$ valor activo ${ }^{U}$

Donde,

$\Delta$ Valor activo $=$ valor generado por la cobertura de costos fijos

Valor activo ${ }^{U}=$ valor activo antes de realizar la cobertura (unhedged)

Valor activo ${ }^{H}=$ valor activo después de realizar la cobertura (unhedge)

$r_{m}=$ retorno del mercado

$r=$ tasa libre de riesgo

Valor activo $^{U}=\sum_{i=1}^{n} \frac{F C L_{i}}{\left(\left.\begin{array}{c}G A O\left(r_{m}-r\right)\left(\sum_{i=1}^{n}\left(\left[\frac{\left[Q_{i}-Q_{i-1}\right]}{\left[Q_{i-1}\right]}-g\right]\right)\left(\% \Delta E B I T_{i}^{M}-E\left[\% \Delta E B I T_{i}^{M}\right]\right)\right) \\ \operatorname{var}\left(\% \Delta E B I T_{i}^{M}\right)\end{array}\right|^{i}\right.}$

Valor activo $^{H}=\sum_{i=1}^{n} \frac{F C L_{i}}{\left.1+r+\frac{\left(r_{m}-r\right)\left(\sum_{i=1}^{n}\left(\left[\frac{\left[Q_{i}-Q_{i-1}\right]}{\left[Q_{i-1}\right]}-g\right]\right)\left(\% \Delta E B I T_{i}^{M}-E\left[\% \Delta E B I T_{i}^{M}\right]\right)\right.}{\operatorname{var}\left(\% \Delta E B I T_{i}^{M}\right)}\right]^{i}}$
Para simplificar, se hacen los siguientes cambios de variables:

$P R=$ prima riesgo de mercado $=r_{m}-r$

$\% \Delta Q_{i}^{*}=\%$ cambio de la demanda no esperada $=\left[\frac{\left[Q_{i}-Q_{i-1}\right]}{\left[Q_{i-1}\right]}-g\right]$

A partir de lo anterior, se llega a que el valor creado por la cobertura es positivo y la diferencia de la ecuación 69 y la ecuación 68. Así, puede observarse claramente que cuando hay costos fijos, GAO > 1, entonces:

Valor $_{\text {activo }}^{H}>$ Valor activo $^{U}$

Por lo tanto:

$\Delta$ Valor activo $>0$

De lo anterior puede concluirse:

a) El riesgo sistemático beta de un activo está determinado por dos variables: los cambios no esperados en la demanda y el grado de apalancamiento operativo.

b) Al realizar una cobertura de costos fijos, se minimiza el grado de apalancamiento operativo, a través de la eliminación de este componente dentro del cálculo del beta.

c) Al eliminar este componente, el beta se reduce, lo que conlleva un aumento en el valor de dicho activo.

d) Este incremento en el valor del activo puede medirse y expresarse a través de la ecuación 67.

\section{DERIVADO REAL DE EBIT}

El derivado de EBIT buscará minimizar el beta y así aumentar el valor del activo subyacente. La función de EBIT se comporta de la siguiente manera:

$E B I T_{t}=S_{t}-C V_{t}-C F$

Para que una empresa no genere pérdidas, su EBIT deberá ser mayor o igual a 0 . El punto en 
que una empresa no genera ni pérdidas ni ganancias operativas puede catalogarse como punto de equilibrio (figura 18), definido así:

$Q_{t}^{*}=\frac{C F}{(P-C)}$

Donde,

$Q_{\vec{t}}=$ cantidad para alcanzar punto de equilibrio

$P-C=$ margen de contribución unitaria

\section{Gráfica 18}

Punto de equilibrio

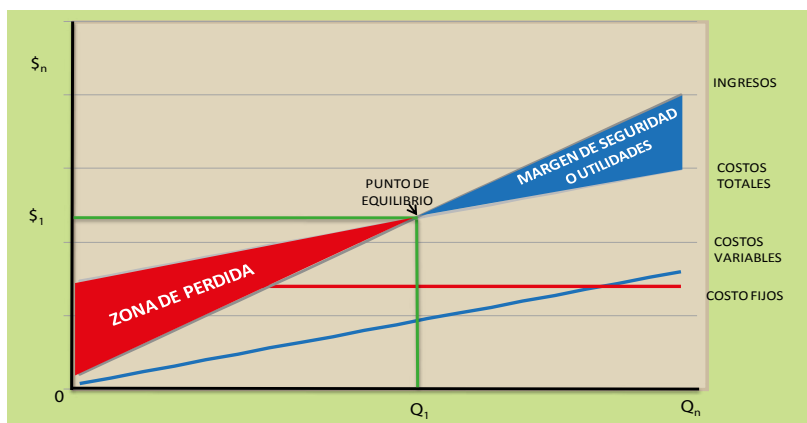

Fuente: elaboración de los autores.

De acuerdo con la anterior función, resulta claro cómo la función de EBIT dependerá de la cantidad demandada en un periodo $t$. Dado que estas dos variables son las que afectan la variabilidad en el EBIT y, por ende, en el beta y en el valor de una empresa o un activo, el objetivo es poder neutralizarlas, para lo cual deben cumplirse dos condiciones: a) que no existan cambios no esperados en la demanda y b) que la estructura de costos replique la estructura de ventas.

La primera puede lograrse con un swap de costos, mientras que la segunda es posible a través de un swap de ventas. Sin embargo, como las ventas se intercambian de variables a fijas, se necesita una estructura de costos que replique unas ventas fijas. Por ello, sería necesario implementar un swap de costos variables (es decir, que convierta costos variables a costos fijos). Como la fórmula de valor presente neto se encuentra en términos discretos, se utilizarán las fórmulas de los swaps en términos discretos:

$S F^{-}=S_{0} \frac{\sum_{i=1}^{t} \frac{(1+g)^{i}}{(1+r)^{i}}}{\sum_{i=1}^{t} \frac{1}{(1+r)^{i}}}$

$C F^{-}=C V_{0} \frac{\sum_{i=1}^{t} \frac{(1+g)^{i}}{(1+r)^{i}}}{\sum_{i=1}^{t} \frac{1}{(1+r)^{i}}}$

Donde,

$S F^{+}=$valor fijo del swap sobre las ventas

$C F^{-}$= valor fijo del swap sobre los costos

$\% \Delta E B I T=\frac{E B I T_{i}^{A}-E B I T_{i-1}^{A}}{E B I T_{i-1}^{A}}=\frac{\left[Q_{i}-Q_{i-1}\right]}{\left[Q_{i-1}\right]} G A O=\frac{\left[\left[S_{t}-S_{t-1}\right]-\left[C V_{t}-C V_{t-1}\right]\right]}{\left[S_{t-1}-C V_{t-1}\right]} G A O$

A partir del swap sobre ventas, y dado que todos los flujos son fijos, puede afirmarse:

$S_{t-1}=S_{t}=S F^{-}$

Adicionalmente, a través del swap sobre costos variables puede determinarse esta ecuación:

$C V_{t-1}=C V_{t}=C F^{-}$

Al substituir en la ecuación 5, se obtiene:

$$
\% \Delta E B I T=\frac{[[0]-[0]]}{\left[S_{0} \frac{\sum_{i=1}^{t} \frac{(1+g)^{i}}{(1+r)^{i}}}{\sum_{i=1}^{t} \frac{1}{(1+r)^{i}}}-C V_{0} \frac{\sum_{i=1}^{t} \frac{(1+g)^{i}}{(1+r)^{i}}}{\sum_{i=1}^{t} \frac{1}{(1+r)^{i}}}\right]} G A O=0
$$

Como el numerador es igual a 0 , que es multiplicado por el grado de apalancamiento operacional, la empresa llega a tener un $\% \triangle E B I T=0$. Así, puede verse claramente que la covarianza del $\% \Delta E B I T$ de este activo con el mercado sería 0 , como sucede con 
la covarianza de cualquier función. De este modo, puede concluirse matemáticamente que el beta desapalancado de este activo sería 0 :

$\beta_{A}=\frac{\operatorname{cov}\left(\% \Delta E B I T_{A}, \% \Delta E B I T_{m}\right)}{\operatorname{var}\left(\% \Delta E B I T_{m}\right)}=\frac{\operatorname{cov}\left(0, \% \Delta E B I T_{m}\right)}{\operatorname{var}\left(\% \Delta E B I T_{m}\right)}=\frac{0}{\operatorname{var}\left(\% \Delta E B I T_{m}\right)}=0$

Al utilizar el modelo CAPM simple, la tasa de descuento se expresa así:

$T D_{i}=k_{A}=r+\left(r_{m}-r\right) \beta A$

Donde,

$k_{A}=$ costo de los activos

$\beta_{U}=$ beta desapalancado

$\beta_{A}=$ beta apalancado

$\beta_{E}=$ beta de equity o patrimonio

Al sustituir el beta de nuestro modelo, se obtiene que la tasa de descuento de los flujos se reduce a la siguiente ecuación:

$T D_{i}=k_{A}=r+\left(r_{m}-r\right) \beta_{A}=r+\left(r_{m}-r\right) 0=r$

$T D_{i}=r$

En la ecuación 12 se resume el impacto de la cobertura sobre la tasa de descuento, es decir, esta se reduce a la tasa libre de riesgo. Para medir el impacto que tiene un swap EBIT sobre el valor de una empresa o un activo, se utiliza el mismo mecanismo que para determinar el derivado de costos:

$\Delta$ Valor activo $=$ Valor activo $^{H}-$ Valor activo $^{U}$

[84]

Valor activo ${ }^{U}=\sum_{i=1}^{n} \frac{F C L_{i}}{\left[1+r+\frac{G A O(P R)\left(\sum_{i=1}^{n}\left(\% \Delta Q_{i}^{*}\right)\left(\% \Delta E B I T_{i}^{M}-E\left[\% \Delta E B I T_{i}^{M}\right]\right)\right)}{\operatorname{var}\left(\% \Delta E B I T_{i}^{M}\right)}\right]^{i}}$

Valor $\operatorname{activo}^{H}=\sum_{i=1}^{n} \frac{F C L_{i}}{[1+r]^{i}}$

$\Delta$ Valor activo $=\sum_{i=1}^{n} \frac{F C L_{i}}{[1+r]^{i}}-\frac{F C L_{i}}{\left[1+r+\frac{G A O(P R)\left(\sum_{i=1}^{n}\left(\% \Delta Q Q_{i}^{*}\right)\left(\% \Delta E B I T_{i}^{M}-E\left[\% \Delta E B I T T_{i}^{M}\right)\right)\right]^{i}}{\operatorname{var}\left(\% \Delta E B I T_{i}^{M}\right)}\right.}$
La anterior ecuación es la que refleja el valor creado por el derivado real de EBIT. Como se ha demostrado, claramente esta es mayor a 0 . Esto va en directa contradicción de la tesis de Clifford, ya que, como se ha comprobado, las coberturas de riesgo son capaces de la creación del valor a través de la reducción de la tasa de descuento, lo cual puede lograrse mediante una cobertura operacional de la estructura de utilidad operacional generada.

\section{SIMULACIÓN DEL DERIVADO DE EBIT}

Para comprobar lo anterior, se llevó a cabo una simulación desde la cual se realizaron los respectivos swaps, para luego medir el impacto en el valor del activo. A partir de la simulación, se tomaron los siguientes supuestos, en función de facilitar los cálculos:

a) No hay depreciaciones o amortizaciones de activos fijos intangibles.

b) No hay impuestos.

c) No hay inversiones adicionales en capital de trabajo.

d) No hay inversiones adicionales en Capex (bienes de capitales).

De esta forma, el flujo de caja libre es igual al EBIT. Por espacio, solo se muestran los primeros treinta datos de la simulación (tablas 4-7).

Tabla 4

Datos de entrada en el modelo de simulación

\begin{tabular}{|c|c|}
\hline Dato & Valor \\
\hline P & 500 \\
\hline C & 200 \\
\hline Q0 & 1000 \\
\hline CF & 180.000 \\
\hline r & $10 \%$ \\
\hline rm & $15 \%$ \\
\hline g & $3 \%$ \\
\hline t & 2996 \\
\hline
\end{tabular}

Fuente: elaboración de los autores.

La tabla 5 muestra los datos de la estructura de pérdidas y ganancias proyectada antes de realizarse el swap sobre el EBIT. 
Simulación del swap de EBIT

\begin{tabular}{|c|c|c|c|c|}
\hline $\mathbf{T}$ & $\mathrm{E}(\mathrm{St})$ (ventas variables) & $\begin{array}{l}\mathrm{E}(\mathrm{CVt}) \text { (costos } \\
\text { variables) }\end{array}$ & $E(C F)$ (costos fijos) & E (EBIT) (EBIT variable) \\
\hline 0 & 500 & 200 & 180 & 120 \\
\hline 1 & 515 & 206 & 180 & 129 \\
\hline 2 & 530.450 & 212.180 & 180 & 138.270 \\
\hline 3 & 546.364 & 218.545 & 180 & 147.818 \\
\hline 4 & 562.754 & 225.102 & 180 & 157.653 \\
\hline 5 & 579.637 & 231.855 & 180 & 167.782 \\
\hline 6 & 597.026 & 238.810 & 180 & 178.216 \\
\hline 7 & 614.937 & 245.975 & 180 & 188.962 \\
\hline 8 & 633.385 & 253.354 & 180 & 200.031 \\
\hline 9 & 652.387 & 260.955 & 180 & 211.432 \\
\hline 10 & 671.958 & 268.783 & 180 & 223.175 \\
\hline 11 & 692.117 & 276.847 & 180 & 235.270 \\
\hline 12 & 712.880 & 285.152 & 180 & 247.728 \\
\hline 13 & 734.267 & 293.707 & 180 & 260.560 \\
\hline 14 & 756.295 & 302.518 & 180 & 273.777 \\
\hline 15 & 778.984 & 311.593 & 180 & 287.390 \\
\hline 16 & 802.353 & 320.941 & 180 & 301.412 \\
\hline 17 & 826.424 & 330.570 & 180 & 315.854 \\
\hline 18 & 851.217 & 340.487 & 180 & 330.730 \\
\hline 19 & 876.753 & 350.701 & 180 & 346.052 \\
\hline 20 & 903.056 & 361.222 & 180 & 361.833 \\
\hline 21 & 930.147 & 372.059 & 180 & 378.088 \\
\hline 22 & 958.052 & 383.221 & 180 & 394.831 \\
\hline 23 & 986.793 & 394.717 & 180 & 412.076 \\
\hline 24 & 1.016 .397 & 406.559 & 180 & 429.838 \\
\hline 25 & 1.046 .889 & 418.756 & 180 & 448.133 \\
\hline 26 & 1.078 .296 & 431.318 & 180 & 466.977 \\
\hline 27 & 1.110 .645 & 444.258 & 180 & 486.387 \\
\hline 28 & 1.143 .964 & 457.586 & 180 & 506.378 \\
\hline 29 & 1.178 .283 & 471.313 & 180 & 526.970 \\
\hline 30 & 1.213 .631 & 485.452 & 180 & 548.179 \\
\hline
\end{tabular}

Fuente: elaboración de los autores. 
Tabla 6

Resultados del swap de EBIT

\begin{tabular}{|c|c|c|c|c|c|}
\hline $\mathrm{T}$ & Ventas fijas & Costos fijos & Costos fijos & EBIT fijo & $\% \Delta$ EBIT \\
\hline 0 & 714.286 & 285.714 & 180 & 248.571 & \\
\hline 1 & 714.286 & 285.714 & 180 & 248.571 & 0,00 \\
\hline 2 & 714.286 & 285.714 & 180 & 248.571 & 0,00 \\
\hline 3 & 714.286 & 285.714 & 180 & 248.571 & 0,00 \\
\hline 4 & 714.286 & 285.714 & 180 & 248.571 & 0,00 \\
\hline 5 & 714.286 & 285.714 & 180 & 248.571 & 0,00 \\
\hline 6 & 714.286 & 285.714 & 180 & 248.571 & 0,00 \\
\hline 7 & 714.286 & 285.714 & 180 & 248.571 & 0,00 \\
\hline 8 & 714.286 & 285.714 & 180 & 248.571 & 0,00 \\
\hline 9 & 714.286 & 285.714 & 180 & 248.571 & 0,00 \\
\hline 10 & 714.286 & 285.714 & 180 & 248.571 & 0,00 \\
\hline 11 & 714.286 & 285.714 & 180 & 248.571 & 0,00 \\
\hline 12 & 714.286 & 285.714 & 180 & 248.571 & 0,00 \\
\hline 13 & 714.286 & 285.714 & 180 & 248.571 & 0,00 \\
\hline 14 & 714.286 & 285.714 & 180 & 248.571 & 0,00 \\
\hline 15 & 714.286 & 285.714 & 180 & 248.571 & 0,00 \\
\hline 16 & 714.286 & 285.714 & 180 & 248.571 & 0,00 \\
\hline 17 & 714.286 & 285.714 & 180 & 248.571 & 0,00 \\
\hline 18 & 714.286 & 285.714 & 180 & 248.571 & 0,00 \\
\hline 19 & 714.286 & 285.714 & 180 & 248.571 & 0,00 \\
\hline 20 & 714.286 & 285.714 & 180 & 248.571 & 0,00 \\
\hline 21 & 714.286 & 285.714 & 180 & 248.571 & 0,00 \\
\hline 22 & 714.286 & 285.714 & 180 & 248.571 & 0,00 \\
\hline 23 & 714.286 & 285.714 & 180 & 248.571 & 0,00 \\
\hline 24 & 714.286 & 285.714 & 180 & 248.571 & 0,00 \\
\hline 25 & 714.286 & 285.714 & 180 & 248.571 & 0,00 \\
\hline 26 & 714.286 & 285.714 & 180 & 248.571 & 0,00 \\
\hline 27 & 714.286 & 285.714 & 180 & 248.571 & 0,00 \\
\hline 28 & 714.286 & 285.714 & 180 & 248.571 & 0,00 \\
\hline 29 & 714.286 & 285.714 & 180 & 248.571 & 0,00 \\
\hline 30 & 714.286 & 285.714 & 180 & 248.571 & 0,00 \\
\hline
\end{tabular}

Fuente: elaboración de los autores. 
Después de la cobertura, la estructura de pérdidas y ganancias queda como se muestra en la tabla 6.

A partir de lo anterior, se simuló el valor antes y después de la cobertura, para hallar el valor creado por el swap sobre el EBIT (tabla 7).

Tabla 7

Resultados del swap del EBIT

\begin{tabular}{|c|c|}
\hline Modelo & Resultado \\
\hline EBIT fijo* $^{*}$ & 285.714 \\
\hline $\mathrm{V}(\mathrm{A}) \mathrm{u}$ (unhedged) & 1.375 .000 \\
\hline $\mathrm{V}(\mathrm{A}) \mathrm{h}$ (hedged) & 2.485 .714 \\
\hline$\Delta \mathrm{V}(\mathrm{A})$ (valor creado por cobertura) & 1.110 .714 \\
\hline
\end{tabular}

Fuente: elaboración de los autores.

\section{CONCLUSIONES}

La cobertura sobre el EBIT elimina los cambios no esperados en la demanda, a través de una combinación de swap de ventas y swap de costos variables. El swap de EBIT ocasiona que este último pase de ser un EBIT con una alta variabilidad a ser uno con una variabilidad nula. Este derivado se puede contemplar así:

Swap EBIT - Swap ventas + Swap costos variables

Al implementar este instrumento, el riesgo sistemático del activo (beta) se convierte en 0 , lo cual lleva a que la tasa de descuento se convierta en una tasa libre de riesgo. En teoría, podría conceptualizarse como un activo sin riesgo.

Lo anterior no quiere decir que el riesgo no exista, sino que el efecto neto de las posiciones tomadas lleva a que no exista variabilidad en los flujos de caja del EBIT; por ende, su apropiada tasa de descuento deberá ser la tasa libre de riesgo.

A pesar de que se elimina la variabilidad en el EBIT (como si se eliminara el riesgo), los flujos de caja esperados son los mismos con el valor proyectado de las oportunidades de crecimiento.
Como el beta se convierte en 0 , la tasa de descuento se minimiza y, por lo tanto, se maximiza el valor de la empresa. La gráfica 19 muestra el valor de un activo en función de su beta desapalancado.

Gráfica 19

Efecto sistemático sobre el valor de la empresa

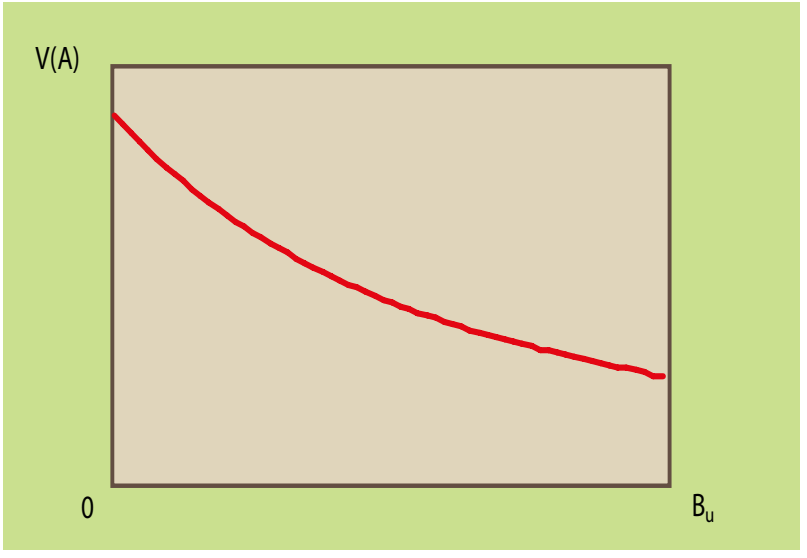

Fuente: elaboración de los autores.

Hacer un swap de EBIT es el nivel óptimo de maximización de valor de un activo, dados unos flujos de caja proyectados.

Una alta volatilidad en el EBIT aumenta la probabilidad de quiebra. Esto puede definirse así:

$E_{t}=E_{t-1}+U_{t}-d i v_{t}$

Donde,

$E_{t}=$ valor del patrimonio en el tiempo $t$

$E_{t-1}=$ valor del patrimonio en el tiempo $t-1$

$U_{t}=$ utilidades del ejercicio en el tiempo $t$

$d i v_{t}=$ dividendos distribuidos a los accionistas en $t$

Por lo tanto, entre mayor sea la probabilidad de que el EBIT tome valores altamente negativos, mayor será la probabilidad de que el valor del patrimonio sea negativo. Si se asume que no se distribuyen dividendos (es absolutamente lógico pensar que cuando se producen pérdidas en el ejercicio no existirán distribuciones de dividendos), el patrimonio será negativo en tanto 
$U_{t}+E_{t}-1<0$. Sin embargo, como indican Cruz, Vargas y Vargas (2009), una empresa puede tener un valor negativo del patrimonio y no entrar en default (suspensión de pagos), lo cual es debido a la estructura de sus pasivos de corto y largo plazo. Se sostiene que una empresa llegará a default cuando valor activos ${ }_{t}-$ el punto de default $=0$. Según lo anterior, puede concluirse que entre mayor sea la varianza del EBIT, mayor será la probabilidad de que el valor de los activos de una empresa llegue a cruzar el punto de default y, por ende, entre en quiebra. Por consiguiente, una cobertura sobre el EBIT reduce la probabilidad de quiebra de una empresa. Además, al reducir esta probabilidad, también se minimizan los costos de transacción de esta.

Existen debates acerca del posible efecto que puede tener la probabilidad de quiebra de una empresa sobre el beta de la deuda, específicamente cuando se encuentra con altos niveles de apalancamiento. Sin embargo, está comprobado que los efectos de los costos de transacción involucrados en un proceso de quiebra, sean estos de una naturaleza legal, administrativa o jurídica, pueden ser significativos. Evidencia empírica puede observarse en los papers de Warner (1977) o Weiss (1990), mientras que Smith y Stultz (1985) utilizaban el mismo argumento para justificar la necesidad de reducir la volatilidad. El impacto que tiene una cobertura del EBIT sobre la probabilidad de quiebra de una empresa puede representarse a través de un movimiento paralelo de la curva de distribución del valor de la empresa y a través de una curva con un mayor coeficiente de curtosis, ya que al disminuirse la varianza del EBIT, un mayor porcentaje de datos se concentran sobre el valor esperado. La gráfica 20 muestra el efecto que tiene una cobertura a través del costo de quiebra sobre el valor de una empresa.

Como puede apreciarse, en la curva existe una reducción en la probabilidad de quiebra de la empresa (la que pasa del área gris al área negra). A la vez, esto conlleva un desplazamiento de la distribución: el valor esperado del valor del activo pasa de E1 (V) a E2 (V). Sin embargo, Tekavčič,
Gráfica 20

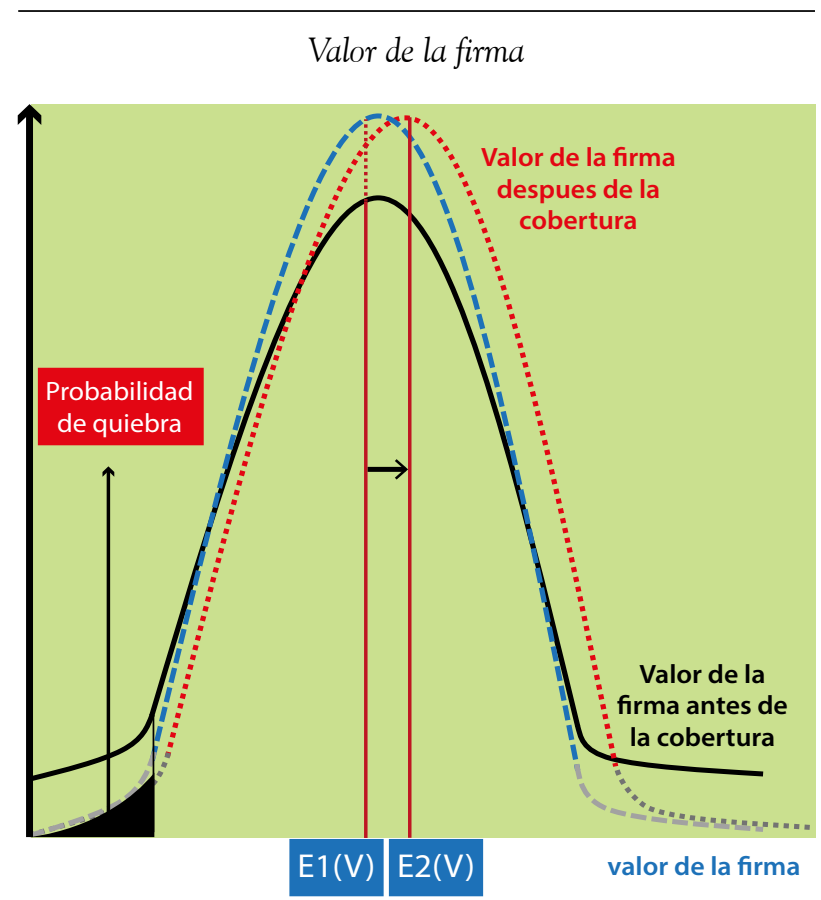

Fuente: Bartram (2000).

Šević y Sprčić (2008) exponen que mientras la reducción del costo de quiebra aumenta el valor de una empresa, también incrementa aún más el valor del patrimonio (o valor de las acciones), ya que aumenta el potencial para llevar deuda. Esto ocurre a causa del ahorro tributario generado por el efecto de impuestos sobre la deuda.

Como se mencionó, los impuestos son una de las formas en que la cobertura puede afectar el valor de un activo. Así, dado que la deuda genera un ahorro tributario que aumenta el valor de un activo, sería más conveniente financiar una empresa a través de deuda. Sin embargo, al sobrepasar ciertos niveles de deuda, las empresas comienzan a crear costos de transacción que se ven reflejados en su mayor probabilidad de quiebra. Por lo tanto, será conveniente seguir aumentando el nivel de apalancamiento financiero de una firma, siempre que el cambio marginal (primera derivada) en el efecto del ahorro tributario sea mayor que el cambio marginal (primera derivada) de los costos de quiebra. Lo anterior nos lleva a concluir que una disminución en la probabilidad de quiebra de una 
Gráfica 21

Apalancamiento de la firma

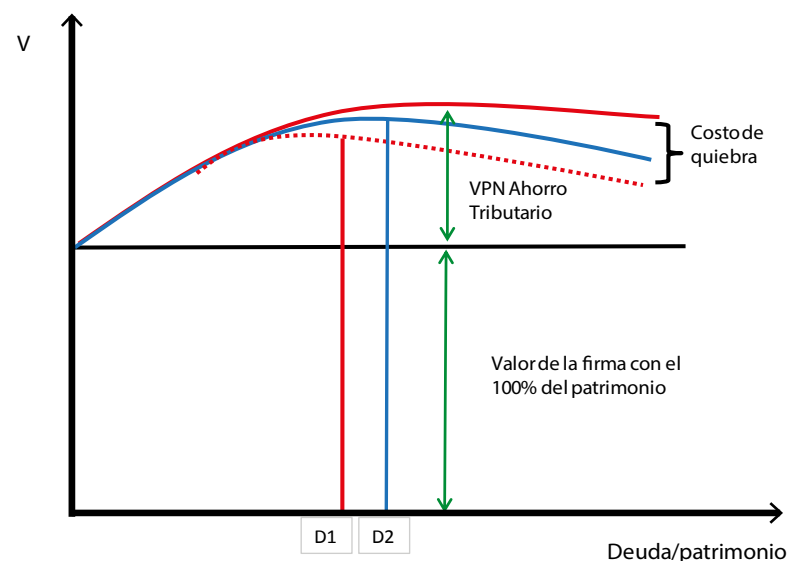

Fuente: Bartram (2000). empresa llevaría a una disminución en el cambio marginal (primera derivada) de los costos de quiebra y, por ende, generaría un desplazamiento del nivel óptimo de apalancamiento financiero de la empresa. Ello permitiría que una empresa pueda aumentar su nivel óptimo de apalancamiento e incrementar el valor generado por el escudo o ahorro tributario a partir de ese mayor apalancamiento, lo cual claramente conllevaría un mayor valor del activo (gráfica 21 ).

La única forma como una empresa puede aumentar el valor de sus activos es incrementando el valor esperado de sus flujos de caja. Lo anterior puede lograrse a través de nuevos proyectos reales (investment policy).

\section{REFERENCIAS}

1. Bartram, S. M. (2000). Corporate risk management as a lever for shareholder value creation. FinancialMarkets, Institutions and Instruments, 9, 279-324.

2. Besley, S. y Brigham, E. F. (2001). Fundamentos de administración financiera. México: McGraw-Hill.

3. Black, F. y Scholes, M. S, (1972). The valuation of option contracts and a test of market efficiency. Journal of Finance, 27, 299-417.

4. Cruz, M., Vargas, J. y Vargas, E. (2009). Aproximación de reclamos contingentes, para la predicción de riesgo de crédito en sus medidas de determinación de la distancia de default y su probabilidad de quiebra para Colombia. Estudios Gerenciales, 27(118), 43-66.

5. Damodaran, A. (2002). Investment valuation. Nueva York: John Wiley \& Sons.

6. Damodaran, A. (2008). Strategic risk taking: a framework for risk management. Nueva Jersey: Pearson Education.

7. Fama, E. F. (1970). Efficient capital markets: a review of theory and empirical work. Journal of Finance, $25,383-417$

8. Horngren, C. T., Datar, S. M. y Foster, G. (2007). Contabilidad de costos: un enfoque gerencial. México: Pearson.

9. Keynes, J. M. (1936). The general theory of employment, interest and money. Cambridge: Cambridge University Press.

10. Knight, E. H. (1921). Risk, uncertainty and profit. Nueva York: Hart, Schaffner and Marx.

11. Kulkarni, M., Powers, M. y Shannon, D. (1991). The use of segment earnings betas in the formation of divisional hurdle rates. Journal of Business Finance and Accounting, 18, 497-512.

12. Markowitz, H M. (1952). Portfolio selection. Journal of Finance, 7, 77-91.

13. Merton, R. C. (1973). Theory of rational option pricing. Bell Journal of Economics and Management Science, 4, 141-183. 
14. Modigliani, F. y Merton, H. M. (1958). The cost of capital, corporation finance and the theory of investment. American Economic Review, 48, 261-297.

15. Nash, J. F. (1950). Equilibrium points in N-Person games. Proceedings of the National Academy of Sciences of the United States of America, 36, 48-49

16. Samuelson, P. A. (1947). Foundations of economic analysis. Harvard University Press, 48, 261-297

17. Sharpe, W. F. (1964). Capital asset prices: a theory of market equilibrium under conditions of risk. Journal of Finance, 19, 425-442.

18. Smith, C. W. (1995). Corporate risk management: theory and practice. Journal of Derivatives, 4, 21-30.

19. Smith, C. y Stultz, R. (1985). The determinants of firms' hedging policies. Journal of Financial and Quantitative Analysis, 20, 391-405.

20. Smithson, C. W. (1987). A LEGO approach to financial engineering: an introduction to forwards, futures, swaps and options. Midland Corporate Finance Journal, 4, 16-28.

21. Tekavčič, M., Šević, Ž. y Sprčić, D. (2008). A review of rationales of corporate risk management: fashion or need? International Journal of Economic Sciences and Applied Research, 1, 71-99G.

22. Warner, J. B. (1977). Bankruptcy costs: some evidence. Journal of Finance, 32, 337-347.

23. Weiss, L. A. (1990). Bankruptcy resolution: direct costs and violation of priority of claims. Journal of Financial Economics, 13, 137-151. 